\title{
Donaldson-Witten theory and indefinite theta functions
}

\author{
Georgios Korpas and Jan Manschot \\ School of Mathematics, Trinity College, \\ College Green, Dublin 2, Ireland \\ Hamilton Mathematical Institute, Trinity College, \\ College Green, Dublin 2, Ireland \\ E-mail: george.korpas@maths.tcd.ie, manschot@maths.tcd.ie
}

AbSTRACT: We consider partition functions with insertions of surface operators of topologically twisted $\mathcal{N}=2, \mathrm{SU}(2)$ supersymmetric Yang-Mills theory, or Donaldson-Witten theory for short, on a four-manifold. If the metric of the compact four-manifold has positive scalar curvature, Moore and Witten have shown that the partition function is completely determined by the integral over the Coulomb branch parameter $a$, while more generally the Coulomb branch integral captures the wall-crossing behavior of both Donaldson polynomials and Seiberg-Witten invariants. We show that after addition of a $\overline{\mathcal{Q}}$-exact surface operator to the Moore-Witten integrand, the integrand can be written as a total derivative to the anti-holomorphic coordinate $\bar{a}$ using Zwegers' indefinite theta functions. In this way, we reproduce Göttsche's expressions for Donaldson invariants of rational surfaces in terms of indefinite theta functions for any choice of metric.

Keywords: Supersymmetric Gauge Theory, Topological Field Theories, Supersymmetry and Duality

ARXIV EPRINT: 1707.06235 


\section{Contents}

1 Introduction $\quad 1$

2 Seiberg-Witten geometry $\quad 4$

2.1 The Seiberg-Witten solution 4

2.2 The topologically twisted theory 6

3 The $u$-plane integral $\quad 9$

3.1 The topologically twisted path integral $\quad 9$

$\begin{array}{ll}3.2 \text { Modular invariance of the integrand } & 11\end{array}$

4 Evaluation of the $u$-plane integral $\quad 12$

$\begin{array}{lll}\text { 4.1 The } u \text {-plane integrand as a total derivative } & 13\end{array}$

$\begin{array}{ll}4.2 & \text { Application to the Hirzebruch surfaces } \mathbb{F}_{\ell} \\ \end{array}$

$\begin{array}{lll}4.3 & \text { Application to the projective plane } \mathbb{P}^{2} & 16\end{array}$

5 On the extension to gauge groups with rank $>1 \quad 18$

6 Conclusion and discussion $\quad 20$

$\begin{array}{ll}\text { A Modular forms and theta functions } & 20\end{array}$

B Indefinite theta functions for uni-modular lattices of signature $(1, n-1) 23$

$\begin{array}{ll}\text { C Integrating over the fundamental domain } & 25\end{array}$

\section{Introduction}

Supersymmetric gauge theories with $\mathcal{N}=2$ and $\mathcal{N}=4$ supersymmetry in four dimensions have been a useful and powerful tool for advances in both physics and mathematics. One of the major successes of such quantum field theories is that their path integral (or partition function) may be exactly evaluated. See for a small sample of the large literature [1-7]. A seminal result of Witten [8] prior to these remarkable developments, is the construction of topological quantum field theories by topological twisting of a supersymmetric field theory. Once evaluated on a smooth compact four-manifold $M$ these theories become of cohomological nature and provide topological invariants of $M$. These topological invariants are typically based on moduli spaces of instantons $[9,10]$, which are solution spaces to the anti-self-duality equations $* F= \pm F$ of the Yang-Mills field strength $F$.

We consider in this article the path integral of topologically twisted $\mathcal{N}=2, \mathrm{SU}(2)$ and $\mathrm{SO}$ (3) Yang-Mills theory on a compact four-manifold $M$ without boundary, the DonaldsonWitten theory for short [8]. The full path integral $\mathcal{Z}_{\text {DW }}$ of this theory can be decomposed 
in to a continuous integral $\mathcal{Z}_{u}$ over the Coulomb branch of the theory, where the gauge group is spontaneously broken to $\mathrm{U}(1)$ by a non-vanishing expectation value of the order parameter $u=\frac{1}{16 \pi^{2}}\left\langle\operatorname{Tr} \phi^{2}\right\rangle$, and a contribution ${ }^{1} \mathcal{Z}_{\mathrm{SW}}$ from the points where the effective theory on the Coulomb branch becomes singular $[2,3,11,12]$. Schematically, we then have

$$
\mathcal{Z}_{\mathrm{DW}}=\mathcal{Z}_{\mathrm{SW}}+\mathcal{Z}_{u}
$$

The $u$-plane integral $\mathcal{Z}_{u}$ only contributes for manifolds with $b_{2}^{+}=1$. For this class of four-manifolds, the path integral is not quite a topological invariant, but only piecewise constant as a function of the metric. The metric dependence is captured by the integral over the Coulomb branch $\mathcal{Z}_{u}$.

More accurately, the partition function of Donaldson-Witten theory vanishes without the insertion of additional operators. Since the theory is topological, these operators correspond to (co)homology classes of $M$. To this end, assume $M$ is simply connected and let $p \in$ $H_{0}(M, \mathbb{Q}) \cong \mathbb{Q}$ and $\boldsymbol{x} \in H_{2}(M, \mathbb{Q})$. Then one can consider the following correlation function

$$
\left\langle e^{p \mathcal{O}^{(0)}+\int_{\boldsymbol{x}} \mathcal{O}^{(2)}}\right\rangle
$$

where $\mathcal{O}^{(0)}$ and $\mathcal{O}^{(2)}$ are a point and surface operator, which are discussed in more detail in section 2.2. A famous aspect of Donaldson-Witten theory is that the correlation function (1.2) is a generating function of Donaldson polynomials defined in mathematics $[9,10]$, where they play an important role in the classification of smooth four-manifolds. The Donaldson polynomials $P_{c_{1}, k}(p, \boldsymbol{x}) \in \mathbb{Q}[p, \boldsymbol{x}]$ are polynomials on the rational homology of $M$, $P_{c_{1}, k}: H_{*}(M, \mathbb{Q}) \rightarrow \mathbb{Q}$ and are defined using the geometry of the moduli space $\mathcal{M}_{c_{1}, k}$ of $k$ instantons with first Chern class $c_{1}(E)=\frac{i}{2 \pi} \operatorname{Tr} F .^{2}$ For the mathematical definition of Donaldson invariants, one considers the map $\mu_{D}$, which maps a cycle $\beta \in H_{i}(M, \mathbb{Q})$ to a cocycle $\mu_{D}(\beta) \in H^{4-i}\left(\mathcal{M}_{c_{1}, k}, \mathbb{Q}\right)$, that is a $(4-i)$-form on $\mathcal{M}_{c_{1}, k}$ of the corresponding vector bundle. Restricting to a simply connected closed four-manifold $M$ and letting $p \in H_{0}(M, \mathbb{Q})$ and $\boldsymbol{x} \in H_{2}(M, \mathbb{Q})$, the Donaldson polynomial is defined as the following integral over $\mathcal{M}_{c_{1}, k}$,

$$
P_{c_{1}, k}(p, \boldsymbol{x})=\sum_{r, s \geq 0} \int_{\mathcal{M}_{c_{1}, k}} \mu_{D}(p)^{r} \wedge \mu_{D}(\boldsymbol{x})^{s} .
$$

The integral gives a non-vanishing result only if the degree of the integrand matches the dimension of $\mathcal{M}_{c_{1}, k}$, therefore $2 r+s=\operatorname{dim}_{\mathbb{C}} \mathcal{M}_{c_{1}, k}$ and (1.3) is indeed a polynomial. The correlation function (1.2) equals the sum of (1.3) over $k$ [8]

$$
\Phi_{\boldsymbol{\mu}}(p, \boldsymbol{x})=\sum_{k} P_{c_{1}, k}\left(e^{p}, e^{\boldsymbol{x}}\right),
$$

where $\boldsymbol{\mu} \in \frac{1}{2} c_{1}+H^{2}(M, \mathbb{Z})$ equals half the Stiefel-Whitney class of the bundles.

\footnotetext{
${ }^{1}$ We use the subscript "SW", since this contribution is fully determined by the so-called Seiberg-Witten invariants of $M[11]$.

${ }^{2}$ In fact, $P_{c_{1}, k}(p, \boldsymbol{x})$ only depends on the second Stiefel-Whitney class $w_{2}=c_{1} \bmod H^{2}(M, 2 \mathbb{Z})$ of the associated vector bundle.
} 
The work of Göttsche [13] and Göttsche-Zagier [14] connected Donaldson invariants to the subject of modular forms, which is at first sight rather distant from the above. Göttsche and Zagier realized that Donaldson invariants of rational surfaces, as determined earlier for example in [15-17], could be written as a residue of a combination of modular forms and so-called indefinite theta functions. The latter enjoy much scientific interest in recent years, in part due to their connection to Ramanujan's mock theta functions $[18,19]$. An indefinite theta function $\Theta: \mathbb{H} \rightarrow \mathbb{C}$ is a holomorphic $q$-series defined as a sum over an indefinite lattice $\Lambda$ with signature $(1, n-1)$. The sum is convergent, since the sum is restricted to lattice points with negative definite norm (for the convention taken in this paper). However, $\Theta$ does not transform as a modular form under $\operatorname{SL}(2, \mathbb{Z})$ transformations. The latter can however be cured thanks to the seminal work of Zwegers': one may add a specific non-holomorphic function $R$ to $\Theta$ such that the sum $\widehat{\Theta}=\Theta+R$ transforms as a modular form. Interestingly, the $\bar{\tau}$-derivative $\Psi=\partial_{\bar{\tau}} \widehat{\Theta}$, turns out to be a Siegel-Narain theta function associated to $\Lambda$, whose modular properties are more easily determined using the familiar Poisson resummation.

Let us now return to the physical $u$-plane integral to explain the main result of this paper. The integral can be expressed as an integral over the fundamental domain $\mathbb{H} / \Gamma^{0}(4)$, where $\Gamma^{0}(4) \in \operatorname{SL}(2, \mathbb{Z})$ is the electric-magnetic duality group, after a change of variables from $u$ to the effective coupling constant $\tau$. Subsequently the technique of "lattice reduction" can be applied to evaluate the integral when $b_{2}(M)>1[2,3]$. This technique was originally developed in the context of one loop amplitudes in string theory [20, 21] and also has major mathematical applications [22]. For the manifold $\mathbb{P}^{2}$, with $b_{2}\left(\mathbb{P}^{2}\right)=1$, the integrand was realized as a total derivative with respect to $\bar{\tau}$ using Zagier's modular completion of the class number generating function [23].

The $u$-plane integral provided in this way a physical derivation of the results on Donaldson invariants by Göttsche and Zagier [13, 14], and reproduced in particular generating functions for rational surfaces including $\mathbb{P}^{1} \times \mathbb{P}^{1}$, the wall-crossing formula and the blowup formula [2]. Later work by Griffin, Malmendier and Ono proved agreement [24-26] in additional cases including $\mathbb{P}^{2}$.

The present paper demonstrates that the $u$-plane integral can be evaluated quite generally by expressing it as a total derivative of an indefinite theta function. To this end, we add a $\overline{\mathcal{Q}}$-exact term to the effective action of the Donaldson-Witten theory used in [2], which does not modify the value of the integral by the usual rules of topological field theory. Using techniques of indefinite theta functions developed by Zwegers [18], we show that the modified integrand is a total $\bar{\tau}$-derivative for an arbitrary four-manifold with $b_{2}^{+}=1$. The integrand of the $u$-plane integral equals what is known as the "shadow" of the indefinite theta series (up to an overall multiplicative function). As a result, the $u$-plane integral can be immediately evaluated for a generic choice of metric, and reproduces precisely Göttsche's results for complex algebraic manifolds. The same technique can be applied when matter is included, and we hope similar techniques can be developed for gauge groups with rank larger than one, theories of class $\mathcal{S}[27,28]$ or more general non-Lagrangian theories. We moreover expect that it may be applied more widely as an alternative for "lattice reduction" to evaluate modular integrals. 
From a more general point of view, it is interesting to note that the Coulomb branch integral provides both the holomorphic and non-holomorphic terms of the indefinite theta function. In other cases where such mock modular forms appear in physics, such as in Vafa-Witten theory [1], $\mathrm{AdS}_{3}$ gravity [29], black holes [30-32], or the moonshine phenomenon [33], the holomorphic part has usually the clearest physical interpretation, whereas the non-holomorphic term is typically less well understood.

We conclude the introduction with an outline of the article. For a self-contained presentation, section 2 reviews relevant aspects of Seiberg-Witten theory, and section 3 reviews the path integral of the topologically twisted theory. Section 3 also discusses the $\overline{\mathcal{Q}}$-exact surface operator, and how it modifies the integrand of the $u$-plane integral. In section 4, we evaluate the integral and provide explicit results for a few complex rational surfaces. In section 5 we discuss the generalization of $\overline{\mathcal{Q}}$-exact surface operator for higher rank gauge groups and how it modifies the integrand of the Coulomb branch integral. We conclude with a brief discussion and concluding remarks. The appendices contain relevant properties of modular forms and (indefinite) theta functions.

\section{Seiberg-Witten geometry}

We begin our discussion with a brief reminder of the solution of Seiberg and Witten of the $\mathrm{SU}(2) \mathcal{N}=2$ super Yang-Mills gauge theory on $\mathbb{R}^{4}[34,35]$ (see [36-39] for a review, [40, 41] for a more modern perspective, and $[42,44]$ for a more mathematically inclined discussion).

\subsection{The Seiberg-Witten solution}

The $\mathcal{N}=2$ vector multiplet contains a scalar field $\phi$, two Weyl fermions, $\lambda, \psi$, and the gauge connection $A$, which are all valued in the adjoint representation of $\mathrm{SU}(2)$. For supersymmetry to be closed off-shell, we include a real auxiliary scalar $V$ and a complex auxiliary scalar $W=W_{1}+i W_{2}$ [39]. The potential of the theory is $V(\phi)=g^{-2} \operatorname{Tr}\left[\phi, \phi^{\dagger}\right]^{2}$ and this is minimized by $\phi=a \sigma_{3}$ up to a gauge transformation. The Weyl group of $\mathrm{SU}(2)$ acts by $a \rightarrow-a$, thus $u=\frac{1}{16 \pi^{2}} \operatorname{Tr} \phi^{2}$ is an invariant quantity and functions as a coordinate on the Coulomb branch moduli space. Quantum-mechanically, we let $u$ be the expectation value

$$
u(a)=\frac{1}{16 \pi^{2}}\left\langle\operatorname{Tr} \phi^{2}\right\rangle .
$$

The Coulomb branch of the quantum moduli space $\mathcal{M}_{\mathrm{C}}$ is isomorphic to $\mathbb{P}^{1} \backslash\left\{\infty, \pm \Lambda^{2}\right\}$ with $\Lambda$ being a dynamically generated scale. At the points $u= \pm \Lambda^{2}$, the effective Coulomb branch theory becomes singular since either the monopole or dyon, which were integrated out in the effective theory, becomes massless.

The BPS states of the theory carry both electric charge $n_{\mathrm{e}}$ and magnetic charge $n_{\mathrm{m}}$ that belong to a charge lattice, $\left(n_{\mathrm{e}}, n_{\mathrm{m}}\right) \in \Gamma_{u} \cong \mathbb{Z} \times \mathbb{Z}$. The complexified mass (or central charge) for a dyonic state is

$$
Z_{u}=n_{\mathrm{e}} a+n_{\mathrm{m}} a_{D} .
$$


At weak coupling $g^{2} \rightarrow 0$, these charges are related to the Coulomb branch parameter $u$ and the effective complexified gauge coupling $\tau=\frac{\theta}{\pi}+\frac{8 \pi i}{g^{2}} \in \mathbb{H}$ by the relations

$$
a(u)=\sqrt{\frac{u}{2}}, \quad a_{D}(u)=\tau a(u) .
$$

In the seminal work by Seiberg and Witten [34, 35], $u, a$ and $a_{D}$ are determined for arbitrary coupling constant $\tau$, making use of an elliptic curve $\Sigma$, whose complex structure is identified with $\tau$. The Coulomb branch corresponds to a family of elliptic curves, which are identified as the solution sets to the following algebraic equation [35]

$$
y^{2}=4 x\left(x^{2}-u x+\frac{1}{4} \Lambda^{4}\right), \quad x, y \in \mathbb{C},
$$

where $\Lambda$ is the dynamically generated mass scale of the theory. One can determine $u$ in terms of $\tau$ using techniques from the theory of elliptic curves. Substituting for $x=$ $\alpha^{-2} \tilde{x}+\frac{1}{3} u$ and $y=\alpha^{-3} \tilde{y}$ brings the curve in Weierstrass form

$$
\tilde{y}^{2}=4 \tilde{x}^{3}-g_{2} \tilde{x}-g_{3}
$$

The constants $g_{2}$ and $g_{3}$ are given in terms of Eisenstein series $E_{k}$ as $g_{2}=\frac{4 \pi^{4}}{3} E_{4}$ and $g_{3}=\frac{8 \pi^{6}}{27} E_{6}$. See Equation (A.3) in appendix A for the definition of the $E_{k}$. The parameter $\alpha$ can be expressed as

$$
\alpha=\frac{\sqrt{2} \pi}{\Lambda} \vartheta_{2} \vartheta_{3}
$$

where $\vartheta_{j}$ are the Jacobi theta functions defined in (A.8) in appendix A. ${ }^{3}$ This gives for $u=u(\tau)$ in terms of Jacobi theta functions

$$
\frac{u}{\Lambda^{2}}=\frac{\vartheta_{2}^{4}+\vartheta_{3}^{4}}{2 \vartheta_{2}^{2} \vartheta_{3}^{2}}=\frac{1}{8} q^{-\frac{1}{4}}+\frac{5}{2} q^{\frac{1}{4}}-\frac{31}{4} q^{\frac{3}{4}}+O\left(q^{\frac{5}{4}}\right)
$$

where $q=e^{2 \pi i \tau}$. The function $u$ is left invariant by a congruence subgroup of the full modular group $\mathrm{SL}(2, \mathbb{Z})$. Using the transformation properties of the Jacobi theta functions (A.9), one verifies that the congruence subgroup is $\Gamma^{0}(4)$ of $\mathrm{SL}(2, \mathbb{Z})$ (A.2).

The discriminant $\Delta$ of the curve is proportional to $u^{2}-\Lambda^{4}$, and vanishes or diverges when the curve (2.1) is singular giving three distinct degenerations of the elliptic curve. Physically, a monopole or dyon becomes massless for $\Delta=0$. These singular points should be excluded from the Coulomb branch, and this is the reason which lead us to the identification of $\mathcal{M}_{\mathrm{C}}$ with $\mathbb{P}^{1} \backslash\left\{ \pm \Lambda^{2}, \infty\right\}$ as explained earlier. We may also parametrize the Coulomb branch as a coset of the upperhalf plane, since $u$ is a modular form of $\Gamma^{0}(4)$. Therefore, $\mathcal{M}_{\mathrm{C}}$ can alternatively be identified with $\mathbb{H} / \Gamma^{0}(4)$. This fundamental domain has three cusps for $\tau \rightarrow i \infty, \tau \rightarrow 0$ and $\tau \rightarrow 2$, which correspond respectively to weak coupling, $u=\Lambda^{2}$ and $u=-\Lambda^{2}$.

\footnotetext{
${ }^{3}$ Our conventions are such that for $\Lambda=1, u$ has the same $q$-expansion as in $[2,3,35]$.
} 
Using rigid special geometry, $a_{D}$ is derived from the prepotential $\mathcal{F}$ that specifies the low energy effective action of the theory,

$$
\mathcal{F}(a, \Lambda)=\frac{4 i}{\pi} a^{2} \log \left(\frac{a}{\Lambda}\right)+a^{2} \sum_{k=0}^{\infty} c_{k}\left(\frac{\Lambda}{a}\right)^{4 k} .
$$

The second summand corresponds to instanton corrections. The magnetic dual of $a$ is expressed in terms of $\mathcal{F}$ as

$$
a_{D}=\frac{\partial \mathcal{F}}{\partial a}
$$

and for the complexified gauge coupling we have

$$
\tau=\frac{\partial a_{D}}{\partial a} .
$$

Seiberg and Witten expressed $a$ and $a_{D}$ as integrals of a differential $\lambda$ over one-cycles of the curve $\Sigma$. To this end, let $A$ and $B$ form a symplectic basis of $H_{1}(\Sigma, \mathbb{Z})$. Then $a$ and $a_{D}$ are given as

$$
a=\int_{A} \lambda, \quad a_{D}=\int_{B} \lambda .
$$

The right behavior at the singularities and positivity of the metric imply that the differential $\lambda$ satisfies

$$
\frac{d \lambda}{d u}=\frac{\sqrt{2}}{4 \pi} \frac{d x}{y} \in H^{1}(\Sigma, \mathbb{C})
$$

Choosing a complex coordinate on the curve $\Sigma, z \in \mathbb{C} /\{m \tau+n\}, m, n \in \mathbb{Z}$, we let the $A$-cycle correspond to the straight line connecting 0 to 1 , and the $B$-cycle the straight line from 0 to $\tau$. To express $a$ in terms of modular forms, we use the map of the parametrization (2.1) of the Seiberg-Witten curve to the Weierstrass form. Then $(\tilde{x}, \tilde{y})=\left(\wp(z), \wp^{\prime}(z)\right)$. As a result one finds that

$$
\frac{d a}{d u}=\frac{1}{2 \Lambda} \vartheta_{2} \vartheta_{3}
$$

Integrating with respect to $u$ expresses $a$ in terms of (quasi)-modular forms

$$
\frac{a}{\Lambda}=\frac{2 E_{2}+\vartheta_{2}^{4}+\vartheta_{3}^{4}}{6 \vartheta_{2} \vartheta_{3}}=\frac{1}{4} q^{-\frac{1}{8}}+\frac{3}{2} q^{\frac{3}{8}}-\frac{21}{4} q^{\frac{7}{8}}+O\left(q^{\frac{11}{8}}\right)
$$

See equation (A.3) in appendix A for the definition of $E_{2}$.

\subsection{The topologically twisted theory}

Topological twisting allows to arrive at a topological quantum field theory starting from a theory with extended supersymmetry [8]. After topologically twisting the holonomy group with the $\mathrm{SU}(2)_{R}$ R-symmetry, one of the original supersymmetry generators transforms trivially under the twisted holonomy group of the four-manifold. This generator, denoted by $\overline{\mathcal{Q}}$, is the BRST operator of the theory and all observables are $\overline{\mathcal{Q}}$-closed. Topological twisting changes the representations of the field content under the rotation group. The 
gauge connection $A$ and complex scalar $a$ remain respectively a 1 -form and a scalar, however the fermionic fields are now a 0 -form $\eta, 1$-form $\psi$ and self-dual 2-form $\chi$. The three auxiliary fields $V, W_{1}$ and $W_{2}$ combine to an auxiliary self-dual two form $D$ [2]. The action of $\overline{\mathcal{Q}}$ on these fields is given by

$$
\begin{aligned}
& {[\overline{\mathcal{Q}}, A]=\psi, \quad[\overline{\mathcal{Q}}, a]=0, \quad[\overline{\mathcal{Q}}, \bar{a}]=\sqrt{2} i \eta,} \\
& {[\overline{\mathcal{Q}}, D]=\left(d_{A} \psi\right)_{+}, \quad\{\overline{\mathcal{Q}}, \psi\}=4 \sqrt{2} d a,} \\
& \{\overline{\mathcal{Q}}, \eta\}=0, \quad\{\overline{\mathcal{Q}}, \chi\}=i\left(F_{+}-D\right),
\end{aligned}
$$

where the subscript + indicates the self-dual component of two-form, thus $F_{+}=\frac{1}{2}(F+* F)$. The four-manifolds considered in this paper have $b_{2}^{+}(M)=1$. The self-dual part of the curvature $\left[F_{+}\right] \in H^{2}(M)$ is for such a four-manifold proportional to the self-dual harmonic form $J \in H^{2}(M)$.

For completeness we present the full Lagrangian $\mathcal{L}$ of the twisted theory on $\mathbb{R}^{4}$ which is given by [2]

$$
\begin{aligned}
\mathcal{L}= & \frac{i}{16 \pi}\left(\bar{\tau} F_{+} \wedge F_{+}+\tau F_{-} \wedge F_{-}\right)+\frac{\tau_{2}}{8 \pi} d a \wedge * d \bar{a}-\frac{\tau_{2}}{8 \pi} D \wedge * D \\
& -\frac{1}{16 \pi} \tau \psi \wedge * d \eta+\frac{1}{16 \pi} \bar{\tau} \eta \wedge d * \psi+\frac{1}{8 \pi} \tau \psi \wedge d \chi-\frac{1}{8 \pi} \bar{\tau} \chi \wedge d \psi \\
& +\frac{i \sqrt{2}}{16 \pi} \frac{d \bar{\tau}}{d \bar{a}} \eta \chi \wedge\left(F_{+}+D\right)-\frac{i \sqrt{2}}{2^{7} \pi} \frac{d \tau}{d a} \psi \wedge \psi \wedge\left(F_{-}+D\right) \\
& +\frac{i}{3 \cdot 2^{11}} \frac{d^{2} \tau}{d a^{2}} \psi \wedge \psi \wedge \psi \wedge \psi-\frac{\sqrt{2} i}{3 \cdot 2^{5} \pi}\left\{\overline{\mathcal{Q}}, \chi_{\mu \nu} \chi^{\nu \lambda} \chi_{\lambda}{ }^{\mu}\right\} \sqrt{g} d^{4} x
\end{aligned}
$$

On curved manifolds, the theory contains further couplings to the background curvature, which were first derived by [12] based on the R-symmetry anomaly. On toric four-manifolds, one may derive these terms from gravitational couplings in the Nekrasov partition function $[4,42]$. As we are working in a topologically twisted theory, the terms can only involve the Euler characteristic $\chi(M)$ and signature $\sigma(M)$ of $M$. For the four-manifolds considered in this paper, $\chi(M)+\sigma(M)=4$. These contributions can be gathered in the measure factor which takes the form

$$
\nu(\tau)=8 i\left(u^{2}-1\right) \frac{d \tau}{d u}\left(\frac{\left(\frac{2 i}{\pi} \frac{d u}{d \tau}\right)^{2}}{u^{2}-1}\right)^{\sigma(M) / 8},
$$

where the multiplicative constants are chosen to match the conventions for Donaldson invariants. Using the identity [43]

$$
u^{2}-1=\frac{i}{4 \pi} \frac{d u}{d \tau}\left(\frac{d u}{d a}\right)^{2}
$$

equation (2.7) equals to

$$
\nu(\tau)=-\frac{2^{\frac{3 \sigma(M)}{4}+1}}{\pi}\left(u^{2}-1\right)^{\frac{\sigma(M)}{8}}\left(\frac{d a}{d u}\right)^{\frac{\sigma(M)}{2}-2} .
$$


The observables of the topological theory lie in the $\overline{\mathcal{Q}}$-cohomology. The $k$-form observables $\mathcal{O}^{(k)}$ relevant for Donaldson theory are obtained as a solution to a descent prescription $[2,8]$,

$$
d \mathcal{O}^{(k)}=\left\{\overline{\mathcal{Q}}, \mathcal{O}^{(k+1)}\right\}
$$

The operator obtained by integrating $\mathcal{O}^{(k)}$ over a $k$-cycle is then automatically $\overline{\mathcal{Q}}$-closed. The descent equations can be solved using an operator $K$, satisfying $\{\overline{\mathcal{Q}}, K\}=d$, such that $\mathcal{O}^{(k)}=K^{k} \mathcal{O}^{(0)}$. We choose $\mathcal{O}^{(0)}=\frac{1}{8 \pi^{2}} \operatorname{Tr} \phi(P)^{2}$ with $P$ a point of $M$. In the effective, topological theory $\phi$ is constant over $M$, and therefore $e^{p \mathcal{O}^{(0)}(P)}=e^{2 p u}$. Using the descent procedure, one finds for the surface observable $\mathcal{O}^{(2)}[2,3]$

$$
I(\boldsymbol{x})=\frac{1}{4 \pi^{2}} \int_{\boldsymbol{x}} \operatorname{Tr}\left[\frac{1}{8} \psi \wedge \psi-\frac{1}{\sqrt{2}} \phi F\right]
$$

with $\boldsymbol{x} \in H^{2}(M)$. In the low energy effective theory, $\mathcal{O}^{(0)}$ is given by the Seiberg-Witten solution, $\mathcal{O}^{(0)}=u$, and the surface operator is modified to [2]

$$
\widetilde{I}_{-}(\boldsymbol{x})=\frac{i}{\sqrt{2} \pi} \int_{\boldsymbol{x}} \frac{1}{32} \frac{d^{2} u}{d a^{2}} \psi \wedge \psi-\frac{\sqrt{2}}{4} \frac{d u}{d a}\left(F_{-}+D\right),
$$

where $F$ is field strength of the remaining $\mathrm{U}(1)$ gauge symmetry. To evaluate the $u$-plane integral using indefinite theta functions, we will add to this surface operator a $\overline{\mathcal{Q}}$-exact operator

$$
\widetilde{I}_{+}(\boldsymbol{x})=-\frac{1}{4 \pi} \int_{\boldsymbol{x}}\left\{\overline{\mathcal{Q}}, \frac{d \bar{u}}{d \bar{a}} \chi\right\}
$$

which using (2.5) evaluates to

$$
\widetilde{I}_{+}(\boldsymbol{x})=-\frac{i}{\sqrt{2} \pi} \int_{\boldsymbol{x}} \frac{1}{2} \frac{d^{2} \bar{u}}{d \bar{a}^{2}} \eta \chi+\frac{\sqrt{2}}{4} \frac{d \bar{u}}{d \bar{a}}\left(F_{+}-D\right) .
$$

This term couples to the self-dual part $F_{+}$of $F$, whereas (2.10) involved only $F_{-}$. Further considerations of such modifications of the topological action are discussed in [45].

Finally, the renormalization group flow to the low energy theory gives rise to a contact term $[2,3], G \boldsymbol{x}^{2}$, which is a consequence of the self-intersection of the cycle $\boldsymbol{x}$ appearing in the surface operators. Since the surface operators $\widetilde{I}_{ \pm}$are $\overline{\mathcal{Q}}$-closed, the coefficient $G$ of the contact term is necessarily holomorphic in $u$. It is conveniently expressed in terms of (quasi)-modular forms

$$
G(u)=\frac{1}{24}\left(8 u-E_{2}\left(\frac{d u}{d a}\right)^{2}\right),
$$

where $E_{2}$ is the Eisenstein series of weight 2 defined by eq. (A.3) in the appendix, or as a derivative to $\vartheta_{4}[3]$

$$
G(u)=-\frac{1}{2 \pi i}\left(\frac{d u}{d a}\right)^{2} \partial_{\tau} \log \vartheta_{4} .
$$

If we want to emphasize the dependence of $G$ on $\tau$, we will write sometimes $G(\tau)$ instead of $G(u)$. 


\section{The $u$-plane integral}

The $u$-plane integral is the path integral over the Coulomb branch of topologically twisted $\mathcal{N}=2$ supersymmetric gauge theory with gauge group $\mathrm{SU}(2)$ or $\mathrm{SO}(3)$. In this section we will explicitly describe the $u$-plane integral following mostly [2]. See for an overview also [46]. Let us start with a few comments on the four-manifold $M$, which we choose to be a simply connected $\left(b_{1}=0\right)$ manifold, compact without boundary. Integration on $M$ gives $H^{2}(M, \mathbb{Z})$ naturally the structure of a lattice $\Lambda \cong \mathbb{Z}^{b_{2}}$ with unimodular quadratic form $Q: H^{2}(M) \rightarrow \mathbb{Z}$ and bilinear form $B: H^{2}(M) \times H^{2}(M) \rightarrow \mathbb{Z}$

$$
Q(\boldsymbol{k}) \equiv \boldsymbol{k}^{2} \equiv \int_{M} \boldsymbol{k} \wedge \boldsymbol{k}, \quad B\left(\boldsymbol{k}_{1}, \boldsymbol{k}_{2}\right) \equiv \int_{M} \boldsymbol{k}_{1} \wedge \boldsymbol{k}_{2},
$$

which has signature $\left(b_{2}^{+}, b_{2}^{-}\right)$. We project $\boldsymbol{k}$ to the positive definite and negative definite subspace as explained in appendix A. Viewed as a 2 -form, $\boldsymbol{k}_{+}$is self-dual under the Hodge *-operation, while $\boldsymbol{k}_{-}$is anti-self-dual.

We restrict in the following to four-manifolds with $b_{2}^{+}=1$. The corresponding lattices $\Lambda$ are completely classifield. If $\Lambda$ is odd, the matrix associated to $Q$ can be brought to the diagonal form

$$
\langle 1\rangle+m\langle-1\rangle
$$

with $m=b_{2}-1$. If $\Lambda$ is even, the matrix associated to $Q$ is equivalent to

$$
\left(\begin{array}{ll}
0 & 1 \\
1 & 0
\end{array}\right) \oplus n \Lambda_{E_{8}},
$$

where $\Lambda_{E_{8}}$ denotes the root lattice of the $E_{8}$ group, and $n=\left(b_{2}-2\right) / 8$.

\subsection{The topologically twisted path integral}

The path integral of the effective theory on the Coulomb branch is given by

$$
\Phi_{\boldsymbol{\mu}}^{J}(p, \boldsymbol{x})=\int[\mathcal{D} X] \nu(\tau) e^{-\int_{M} \mathcal{L}+2 p u+\tilde{I}_{-}(\boldsymbol{x})+\tilde{I}_{+}(\boldsymbol{x})+\boldsymbol{x}^{2} G},
$$

where $[\mathcal{D} X]$ stands for the path integral measure of the fields $[\mathcal{D}(A, a, \bar{a}, \eta, \chi, \psi, D)]$. As mentioned in the introduction and will be confirmed in the following, $\Phi_{\mu}^{J}$ depends discontinuously on the metric $g$, and may jump across walls of marginal stability due to the presence of Abelian instantons. The metric dependence of $\Phi_{\boldsymbol{\mu}}^{J}$ is only through the period point $J=J(g)[16]$.

To evaluate the path integral, we start by integrating out $D$. We do so by substituting for $D$ the solution to its equation of motion. From the terms in the action (2.6) involving $D$, together with the $D$-dependent terms in (2.10) and (2.12), one finds

$$
D=-\frac{2 \operatorname{Im}(d u / d a)}{\tau_{2}} \boldsymbol{x}_{+}+\frac{\sqrt{2} i}{4 \tau_{2}} \frac{d \bar{\tau}}{d \bar{a}} \eta \chi
$$


where $\boldsymbol{x}$ denotes the two-form Poincaré dual to the cycle $\boldsymbol{x}$ in (2.10) and (2.12), or equivalently $\int_{\boldsymbol{x}} \omega=\int_{M} \boldsymbol{x} \wedge \omega$ for any 2 -form $\omega$. To simplify notation, we define the variables $\boldsymbol{\rho} \in H^{2}(M, \mathbb{C})$ and $\boldsymbol{b} \in H^{2}(M, \mathbb{R})$ by

$$
\boldsymbol{\rho}=\frac{\boldsymbol{x}}{2 \pi} \frac{d u}{d a}, \quad \boldsymbol{b}=\frac{\operatorname{Im}(\boldsymbol{\rho})}{\tau_{2}} .
$$

After substitution of (3.5) back in the $D$-dependent terms of the Lagrangian and surface operators, they contribute

$$
\exp \left(-2 \pi \tau_{2} \boldsymbol{b}_{+}^{2}+\frac{i \sqrt{2}}{4} \frac{d \bar{\tau}}{d \bar{a}} \int_{M} \boldsymbol{b}_{+} \wedge \eta \chi\right)
$$

to the integrand of the path integral.

Next we integrate over the fermionic fields. To understand the contribution to the $u$-plane integral from these fields it is useful to discuss the scaling of the fields under a rescaling of the metric: $\lim _{t \rightarrow \infty}=t^{2} g_{0}$ for a fixed metric $g_{0} .{ }^{4}$ The scaling dimensions of the zero modes naturally equals their form degree. These equal the scaling dimensions of the quantum fluctuations of the fields, except for $\eta$, whose quantum fluctuation has dimension 2 instead of 0 [2, section 2.3]. Thus we see that the terms of the Lagrangian involving $\eta$ and $\chi$ have scaling dimension larger than four, except when we replace $\eta$ by its zero-mode $\eta_{0}$. Similarly, the term involving $\eta$ in the surface operator (2.12) has dimension 2 if we replace $\eta$ by its zero mode. One can show that the corrections due to the quantum fluctuations of $\chi$ do not survive in the limit $t \rightarrow \infty$, assuming that $b_{1}(M)=0$.

Therefore, the path integral over the fermionic fields is reduced to the integral over zero modes. Collecting the terms involving the zero modes gives

$$
\int\left[d \eta_{0} d \chi_{0}\right] \exp \left(-\frac{\sqrt{2} i}{16 \pi} \frac{d \bar{\tau}}{d \bar{a}} \int_{M} \eta_{0} \chi_{0} \wedge\left(F_{+}-4 \pi \boldsymbol{b}_{+}\right)-\frac{i}{\pi \sqrt{2}} \int_{\boldsymbol{x}} \frac{1}{2} \frac{d^{2} \bar{u}}{d \bar{a}^{2}} \eta_{0} \chi_{0}\right),
$$

Carrying out the integral gives

$$
\int_{M}\left(\frac{\sqrt{2} i}{16 \pi} \frac{d \bar{\tau}}{d \bar{a}}(F-4 \pi \boldsymbol{b})+\frac{i}{\sqrt{2}} \frac{d \bar{\rho}}{d \bar{a}}\right) \wedge \underline{J}
$$

where $\underline{J}$ is the normalization of $J, J / \sqrt{Q(J)}$. This can interestingly be written in a simpler form as a derivative to $\bar{\tau}$,

$$
\frac{\sqrt{\tau_{2}}}{4 \pi} \frac{d \bar{\tau}}{d \bar{a}} \partial_{\bar{\tau}} \sqrt{2 \tau_{2}} B(F+4 \pi \boldsymbol{b}, \underline{J})
$$

where we used the notation (3.1), and $\partial_{\bar{\tau}}$ acts on all terms to its right.

Finally, the photon path integral contains a sum over all fluxes times a factor of $\tau_{2}^{-\frac{1}{2}}[12] .^{5}$ The $\mathrm{U}(1)$ fluxes $[F] / 4 \pi$ lie in a shift of the integer cohomology group $H^{2}(M, \mathbb{Z})$

\footnotetext{
${ }^{4}$ This one-parameter family of metrics belongs to a single chamber.

${ }^{5}$ Here we assumed $b_{1}=0$; for a non-simply connected four-manifold this factor equals $\tau_{2}^{\left(b_{1}-1\right) / 2}$.
} 
by half the second Stiefel-Whitney class $w_{2}(E)$ of the $\mathrm{SU}(2)$ or $\mathrm{SO}(3)$ bundle $E$, since $F$ is the field strength of the unbroken $\mathrm{U}(1)$ gauge group. We introduce the conjugacy class $\boldsymbol{\mu} \in H^{2}(M, \mathbb{Z} / 2 \mathbb{Z})$, such that $w_{2}(E)=2 \boldsymbol{\mu}+H^{2}(M, 2 \mathbb{Z})$ and $\frac{1}{4 \pi}[F] \in H^{2}(M, \mathbb{Z})+\boldsymbol{\mu}$. With $\boldsymbol{k}=\frac{1}{4 \pi}[F]$, the photon path integral can be written concisely in terms of a Siegel-Narain theta series $\Psi_{\boldsymbol{\mu}}^{J}(\tau, \boldsymbol{\rho})$ defined as

$$
\begin{aligned}
\Psi_{\boldsymbol{\mu}}^{J}(\tau, \boldsymbol{\rho})= & \exp \left(-2 \pi \tau_{2} \boldsymbol{b}_{+}^{2}\right) \sum_{\boldsymbol{k} \in \Lambda+\boldsymbol{\mu}} \partial_{\bar{\tau}}\left(\sqrt{2 \tau_{2}} B(\boldsymbol{k}+\boldsymbol{b}, \underline{J})\right) \\
& \times(-1)^{B\left(\boldsymbol{k}, K_{M}\right)} \exp \left(-i \pi \bar{\tau} \boldsymbol{k}_{+}^{2}-i \pi \tau \boldsymbol{k}_{-}^{2}-2 \pi i B\left(\boldsymbol{k}_{+}, \overline{\boldsymbol{\rho}}\right)-2 \pi i B\left(\boldsymbol{k}_{-}, \boldsymbol{\rho}\right)\right),
\end{aligned}
$$

where we identify $\Lambda$ with $H^{2}(M, \mathbb{Z})$. The first exponential on the right hand side of (3.11) is due to (3.7), and the first term after the summation sign due to (3.10) divided by $\sqrt{\tau_{2}}$. We recognize the couplings in the exponent on the second line as due to the classical YangMills action $[12,47]$ and the surface operators $\tilde{I}_{+}$and $\tilde{I}_{-}$. The sign $(-1)^{B\left(\boldsymbol{k}, K_{M}\right)}$, with $K_{M}$ the canonical class of $M$, arises from integrating out the massive fermions in the Coulomb branch of the topologically twisted theory [12].

The path integral with the insertions of the point and surface operator then equals

$$
\Phi_{\boldsymbol{\mu}}^{J}(p, \boldsymbol{x})=\int_{\mathcal{M}_{C}} d a \wedge d \bar{a} \nu(\tau) \frac{d \bar{\tau}}{d \bar{a}} \Psi_{\boldsymbol{\mu}}^{J}(\tau, \boldsymbol{\rho}) e^{2 p u+\boldsymbol{x}^{2} G} .
$$

The integration domain is most naturally stated in terms of $\tau$, rather then $a$. Since the duality group of Seiberg-Witten theory is $\Gamma^{0}(4)$, the domain for $\tau$ is naturally $\mathbb{H} / \Gamma^{0}(4)$. We then arrive at the following modular integral

$$
\Phi_{\boldsymbol{\mu}}^{J}(p, \boldsymbol{x})=\int_{\mathbb{H} / \Gamma^{0}(4)} d \tau \wedge d \bar{\tau} \tilde{\nu}(\tau) \Psi_{\boldsymbol{\mu}}^{J}(\tau, \boldsymbol{\rho}) e^{2 p u+\boldsymbol{x}^{2} G},
$$

where we defined

$$
\tilde{\nu}=\nu \frac{d a}{d \tau}
$$

\subsection{Modular invariance of the integrand}

For completenes we discuss in this subsection invariance of the integrand under the $\Gamma^{0}(4)$ duality group of Seiberg-Witten theory, which is an important consistency requirement for the integrand. Since $d \tau \wedge d \bar{\tau}$ transforms with weight $(-2,-2)$, the integrand in (3.13) must have modular weight $(2,2)$. Let us start with the function $\Psi_{\boldsymbol{\mu}}^{J}(3.11)$ of the integrand. This is an example of a Siegel-Narain theta function. A general form of such theta functions which suits our purposes is given in appendix A, equation (A.11). To compare (3.13) with that equation, we set $\boldsymbol{z}=\boldsymbol{\rho}$ and $\boldsymbol{b}=\operatorname{Im}(\boldsymbol{\rho}) / \tau_{2}$, with $\boldsymbol{\rho}$ as defined in (3.6). Furthermore, we identify $K$ in (A.11) with the canonical class $K_{M}$, which is a characteristic element of $H^{2}(M, \mathbb{Z}){ }^{6}$ The transformation properties of $\Psi_{\boldsymbol{\mu}}^{J}$ under $\mathrm{SL}(2, \mathbb{Z})$ and $\Gamma^{0}(4)$ are given in (A.12)-(A.16).

We see that $\boldsymbol{\rho}$ appears in $\Psi_{\boldsymbol{\mu}}^{J}$ as an elliptic variable. Indeed, since $\frac{d a}{d u}$ from equation (2.3) is a modular form of weight 1 under $\Gamma^{0}(4), \rho$ transforms as an elliptic variable. More

\footnotetext{
${ }^{6}$ This follows for example from the Hirzebruch-Riemann-Roch theorem for a line bundle.
} 
precisely, one verifies using the properties of the Jacobi theta functions (A.9), that $\rho$ transforms under the two generators of $\Gamma^{0}(4)$ as

$$
\boldsymbol{\rho}(\tau+4)=-\boldsymbol{\rho}(\tau), \quad \boldsymbol{\rho}\left(\frac{\tau}{\tau+1}\right)=\frac{\boldsymbol{\rho}(\tau)}{\tau+1} .
$$

Note that this differs by a minus sign from the usual transformation of an elliptic variable under $\tau \rightarrow \tau+4$.

With these transformations, we can determine the action of $\Gamma^{0}(4)$ generators on $\Psi_{\boldsymbol{\mu}}^{J}(\tau, \boldsymbol{\rho})$. Recall that $\boldsymbol{\mu} \in H^{2}(M, \mathbb{Z} / 2)$ in the path integral. Combining (A.14) and (A.16), we find for the generator $\tau \rightarrow \tau+4$ of $\Gamma^{0}(4)$

$$
\left.\Psi_{\boldsymbol{\mu}}^{J}(\tau, \boldsymbol{\rho})\right|_{\tau \mapsto \tau+4}=-\Psi_{\boldsymbol{\mu}}^{J}(\tau, \boldsymbol{\rho}) .
$$

Using (A.15), we derive for the action of the second generator

$$
\left.\Psi_{\boldsymbol{\mu}}^{J}(\tau, \boldsymbol{\rho})\right|_{\tau \mapsto \frac{\tau}{\tau+1}}=(\bar{\tau}+1)^{2}(\tau+1)^{\frac{b_{2}}{2}} \exp \left(-\frac{\pi i \boldsymbol{\rho}^{2}}{\tau+1}+\frac{\pi i}{4} \sigma(M)\right) \Psi_{\boldsymbol{\mu}}^{J}(\tau, \boldsymbol{\rho}),
$$

where we used $K_{M}^{2}=8+\sigma(M)$ for simply connected four-manifolds with $b_{2}^{+}=1$.

Next we discuss the contact term $e^{\boldsymbol{x}^{2} G}$ with $G$ given in (2.13). Due to the special transformations of the weight two Eisenstein series $E_{2}$ given in (A.5), the contact term transforms as follows

$$
e^{\boldsymbol{x}^{2} G(\tau+4)}=e^{\boldsymbol{x}^{2} G(\tau)}, \quad e^{\boldsymbol{x}^{2} G\left(\frac{\tau}{\tau+1}\right)}=e^{\boldsymbol{x}^{2} G(\tau)+\frac{\pi i}{\tau+1} \boldsymbol{\rho}^{2}} .
$$

The remaining term in the integrand is $\tilde{\nu}$. Using the identity

$$
\left(\frac{\left(\frac{2 i}{\pi} \frac{d u}{d \tau}\right)^{2}}{u^{2}-1}\right)^{\frac{1}{8}}=\vartheta_{4}(\tau)
$$

we can write $\tilde{\nu}$ as

$$
\tilde{\nu}(\tau)=-8 i\left(u^{2}-1\right) \frac{d a}{d u} \vartheta_{4}(\tau)^{\sigma(M)} .
$$

If we express further $u$ and $d a / d u$ in terms of Jacobi theta functions and use the transformation properties (A.9) under $\Gamma^{0}(4)$, one finds

$$
\tilde{\nu}(\tau+4)=-\tilde{\nu}(\tau), \quad \tilde{\nu}\left(\frac{\tau}{\tau+1}\right)=(\tau+1)^{2-\frac{b_{2}(M)}{2}} e^{-\frac{\pi i \sigma(M)}{4}} \tilde{\nu}(\tau)
$$

Combining now (3.16), (3.17), (3.18) and (3.21), we conclude that the integrand of (3.13) has indeed weight $(2,2)$ under $\Gamma^{0}(4)$ as required.

\section{Evaluation of the $u$-plane integral}

The previous section reduced the path integral (3.4) to the integral (3.13) over the fundamental domain $\mathbb{H} / \Gamma^{0}(4)$. This domain is the union of six images of the $\operatorname{SL}(2, \mathbb{Z})$ fundamental 
domain, $\mathcal{F}=\mathbb{H} / \mathrm{SL}(2, \mathbb{Z})$. Taking $\mathcal{F}$ as the familiar "key hole" shaped region of the upper half plane extending along the imaginairy axis, we take $\mathbb{H} / \Gamma^{0}(4)$ as the union of this domain and the images under $\tau \rightarrow \tau+1, \tau+2, \tau+3, \tau+4,-1 / \tau$ and $2-1 / \tau$.

Integrals over $\mathcal{F}$ of modular invariant integrands, $d \tau \wedge d \bar{\tau} F$, are well-studied in the literature. They appear for example as inner product on the space of modular forms [48], as one-loop amplitudes in string theory $[20,21,49]$ or in the context of divisors on symmetric spaces [22]. Depending on the integrand, different techniques are available to evaluate the integral. A common approach to evaluate the integral is to "unfold" $\mathcal{F}$ to the strip $\tau \in \mathbb{H}, \tau_{1} \in\left[-\frac{1}{2}, \frac{1}{2}\right]$, either using an Eisenstein or Poincaré series in the integrand, or using the technique of lattice reduction [20-22]. The latter was also originally used for the $u$-plane integral [2].

However, the integral may be evaluated more straightforwardly in special cases. Namely when the integrand $F$ can be expressed as a total derivative with respect to $\bar{\tau}$,

$$
F=\frac{\partial H}{\partial \bar{\tau}}
$$

with $H=H(\tau, \bar{\tau})$ a function which transforms as a modular form of weight 2. As reviewed in appendix $\mathrm{C}$, the integral receives in this case only a contribution from the cusp at $i \infty$, and the final result is

$$
\int_{\mathcal{F}} d \tau \wedge d \bar{\tau} F=[H]_{q^{0}}
$$

An integral $\Phi$ over the fundamental domain for $\Gamma^{0}(4), \mathbb{H} / \Gamma^{0}(4)$, can similarly be expressed as a sum over its three cusps at $i \infty, 0$ and 2 :

$$
\Phi=\Phi_{\infty}+\Phi_{0}+\Phi_{2}
$$

The contribution of the cusp at infinity, $\Phi_{\infty}$, is

$$
\Phi_{\infty}=4[H]_{q^{0}},
$$

which differs by a factor 4 from (4.2) since the arc for large $\tau_{2}$ runs now from $\tau_{1}=3 \frac{1}{2}$ to $\tau_{1}=-\frac{1}{2}$. The contributions from the other two cusps, at $\tau=0$ and 2 , can be mapped to $i \infty$ using the transformation $\tau \rightarrow-1 / \tau$ and $\tau \rightarrow 1 /(2-\tau)$ respectively.

\subsection{The $u$-plane integrand as a total derivative}

In order to evaluate the integral (3.13), it is clear from the above that we can readily evaluate the integral, if we may express the integrand as a total derivative. To this end, we need to find a non-holomorphic modular form $H$ of weight two, which satisfies

$$
\partial_{\bar{\tau}} H=\tilde{\nu} \Psi_{\mu}^{J}
$$

In the remainder of this section, we determine such an $H$ making use of indefinite theta series [18]. This allows us to rederive the Donaldson invariants for Hirzebruch surfaces and the projective plane, and also the wall-crossing formula for $\Phi_{\boldsymbol{\mu}}^{J}$ for an arbitrary simply connected four-manifold $M$ with $b_{2}^{+}=1$. See appendix B for a concise introduction to 
indefinite theta functions. Here we recall the definition of $\widehat{\Theta}_{\mu}^{J J^{\prime}}: \mathbb{H} \times \mathbb{C}^{b_{2}} \rightarrow \mathbb{C}$. The function $\Theta_{\boldsymbol{\mu}}^{J J^{\prime}}$ depends on two parameters $J$ and $J^{\prime}$. As the notation suggests, we identify $J$ with the period point of the metric of $M$ in $H^{2}(M)$. We choose furthermore $J^{\prime} \in H^{2}(M, \mathbb{Z})$ such that $\left(J^{\prime}\right)^{2}=0$ and $B\left(J, J^{\prime}\right)>0$. We deduce from the classification of Lorentzian lattices, eqs. (3.2) and (3.3), that such a vector does indeed exist for any smooth, closed, oriented four-manifold $M$ with $b_{2}^{+}=1$ and $b_{2}>1$. With this choice of parameters, $\Theta_{\boldsymbol{\mu}}^{J J^{\prime}}$ is defined as

$$
\begin{gathered}
\widehat{\Theta}_{\boldsymbol{\mu}}^{J J^{\prime}}(\tau, \boldsymbol{z})=\sum_{\boldsymbol{k} \in \Lambda+\mu} \frac{1}{2}\left(E\left(\sqrt{2 \tau_{2}} B(\boldsymbol{k}+\boldsymbol{b}, \underline{J})\right)-\operatorname{sgn}\left(\sqrt{2 \tau_{2}} B\left(\boldsymbol{k}+\boldsymbol{b}, J^{\prime}\right)\right)\right) \\
\times(-1)^{B\left(\boldsymbol{k}, K_{M}\right)} q^{-\boldsymbol{k}^{2} / 2} e^{-2 \pi i B(\boldsymbol{z}, \boldsymbol{k})},
\end{gathered}
$$

where $E(t): \mathbb{R} \rightarrow[-1,1]$ is a reparametrization of the error function,

$$
E(t)=2 \int_{0}^{t} e^{-\pi u^{2}} d u=\operatorname{Erf}(\sqrt{\pi} t)
$$

and $\underline{J}=J / \sqrt{Q(J)}$ is the normalization of $J$ as before.

Appendix B discusses that $\widehat{\Theta}_{\boldsymbol{\mu}}^{J J^{\prime}}$ transforms as a modular form of weight $b_{2} / 2$ under $\Gamma^{0}(4)$ (B.4), and that its derivative to $\bar{\tau}$ equals

$$
\partial_{\bar{\tau}} \widehat{\Theta}_{\boldsymbol{\mu}}^{J J^{\prime}}(\tau, \boldsymbol{z})=\Psi_{\boldsymbol{\mu}}^{J}(\tau, \boldsymbol{z}),
$$

with $\Psi_{\boldsymbol{\mu}}^{J}(\tau, \boldsymbol{z})$ equal to the sum over U(1) fluxes (3.11). We see from (4.8) that if we set

$$
H=\tilde{\nu}(\tau) \widehat{\Theta}_{\boldsymbol{\mu}}^{J J^{\prime}}(\tau, \boldsymbol{\rho}) e^{2 p u+\boldsymbol{x}^{2} G},
$$

then it satisfies indeed (4.1) with $F$ the integrand of $u$-plane integral (3.13). Note that there is an ambiguity in (4.8) since addition of a holomorphic modular form to $\widehat{\Theta}_{\mu}^{J J^{\prime}}$ does not change the right hand side. This is related to the ambiguity in the choice of $J^{\prime}$. We assume that this ambiguity can be fixed by basic arguments, for example the existence of an "empty" chamber. We will see this later in this section for the Hirzebruch surfaces and the projective plane $\mathbb{P}^{2}$.

Recall from (4.3) that the contributions to the integral are coming from the three cusps. The contribution from the cusp at infinity (4.4) is given by

$$
4\left[\tilde{\nu}(\tau) \Theta_{\boldsymbol{\mu}}^{J J^{\prime}}(\tau, \boldsymbol{\rho}) e^{2 p u+\boldsymbol{x}^{2} G}\right]_{q^{0}},
$$

where $\Theta_{\mu}^{J J^{\prime}}$ is the holomorphic indefinite theta series (B.1), obtained from (4.6) by replacing $E(x)$ by $\operatorname{sgn}(x)$. One first expands in (4.10) in the fugacities $p$ and $\boldsymbol{x}$, and then in $q$. The contributions to the integral from the other cusps follows similarly after transforming $\tau \rightarrow-1 / \tau$ and $1 /(2-\tau)$ in the integrand. If the metric of $M$ has positive scalar curvature, the $u$-plane integral completely determines the Donaldson invariants.

We briefly mention the wall-crossing formula which was earlier derived from the $u$-plane integral in [2]. This formula gives the discontinuous change of $\Phi_{\boldsymbol{\mu}}^{J}$ under the variation of 
a metric with period point $J_{0}$ to one with period point $J_{1} \in H^{2}(M)$. It is clear from the above that the difference $\Delta \Phi_{\boldsymbol{\mu}}^{J_{1} J_{0}}=\Phi_{\boldsymbol{\mu}}^{J_{1}}-\Phi_{\boldsymbol{\mu}}^{J_{0}}$ is given by

$$
\Delta \Phi_{\boldsymbol{\mu}}^{J_{1} J_{0}}(p, \boldsymbol{x})=\int_{\mathbb{H} / \Gamma^{0}(4)} d \tau \wedge d \bar{\tau} \tilde{\nu}\left(\Psi_{\boldsymbol{\mu}}^{J_{1}}-\Psi_{\boldsymbol{\mu}}^{J_{0}}\right) e^{2 p u+\boldsymbol{x}^{2} G} .
$$

The contribution from the cusp at $i \infty$ gives then

$$
\Delta \Phi_{\boldsymbol{\mu}}^{J_{1} J_{0}}(p, \boldsymbol{x})=4\left[\tilde{\nu}(\tau) \Theta_{\boldsymbol{\mu}}^{J_{1} J_{0}}(\tau, \boldsymbol{\rho}) e^{2 p u+\boldsymbol{x}^{2} G}\right]_{q^{0}},
$$

while the contributions of other cusps are cancelled the wall-crossing of Seiberg-Witten invariants [2]. This reproduces Göttsche's wall-crossing formula [13, Theorem 3.3] and the expression of Göttsche-Zagier in terms of an indefinite theta series [14, Corollary 4.3].

\subsection{Application to the Hirzebruch surfaces $\mathbb{F}_{\ell}$}

In this subsection, we specialize the four-manifold $M$ to one of the Hirzebruch surfaces $\mathbb{F}_{\ell}$. A Hirzebruch surface is a fibration $\pi: \mathbb{F}_{\ell} \rightarrow C$ with fibre $f \cong \mathbb{P}^{1}$ over a base $C \cong \mathbb{P}^{1}$. The base and the fibre form a basis for $H^{2}\left(\mathbb{F}_{\ell}, \mathbb{Z}\right)$, in terms of which the canonical class $K_{\ell}$ is expressed as $K_{\ell}=-2 \boldsymbol{C}-(2+\ell) \boldsymbol{f}$. The intersection matrix for $(\boldsymbol{C}, \boldsymbol{f})$ is

$$
Q_{\mathbb{F}_{\ell}}=\left(\begin{array}{cc}
-\ell & 1 \\
1 & 0
\end{array}\right) .
$$

Note in particular that $\boldsymbol{f}$ is an element of $H^{2}\left(\mathbb{F}_{\ell}\right)$ with vanishing norm, $\boldsymbol{f}^{2}=0$. Two Hirzebruch surfaces $\mathbb{F}_{\ell_{1}}$ and $\mathbb{F}_{\ell_{2}}$ are (real) diffeomorphic if $\ell_{1}=\ell_{2} \bmod 2$, while they are complex diffeomorphic only for $\ell_{1}=\ell_{2}$. For more details on Hirzebruch surfaces see for example [50].

To evaluate $\Phi_{\boldsymbol{\mu}}^{J}$ for $\mathbb{F}_{\ell}$ using (4.9), consider the indefinite theta function (4.6) with the quadratic form (4.12) above. We set $J^{\prime}=\boldsymbol{f}$, which is fixed by the fact that no stable bundles exist for metrics with this period point. Indeed for $J=\boldsymbol{f}, \Theta_{\mu}^{J f}$ vanishes. One may show that only the cusp at $\infty$ contributes to the integral for $\mathbb{F}_{\ell}$, and we arrive thus for $\Phi_{\boldsymbol{\mu}}^{J}$ at the following expression

$$
\Phi_{\boldsymbol{\mu}}^{J}(p, \boldsymbol{x})=32 i\left[\left(u^{2}-1\right) \frac{d a}{d u} \Theta_{\boldsymbol{\mu}}^{J \boldsymbol{f}}(\tau, \boldsymbol{\rho}) e^{2 p u+\boldsymbol{x}^{2} G}\right]_{q^{0}} .
$$

We can simplify the expression for $\Theta_{\mu}^{J f}$ and express it as a (generalized) Appell sum. To this end, write $\boldsymbol{k}$ as $\boldsymbol{k}=\boldsymbol{m}+n \boldsymbol{f}$, with $\boldsymbol{m}$ such that

$$
B(\boldsymbol{m}+\boldsymbol{b}, J) / B(\boldsymbol{f}, J) \in[0,1) .
$$

Then $\Theta_{\mu}^{J f}$ takes the form

$$
\begin{aligned}
\Theta_{\boldsymbol{\mu}}^{J \boldsymbol{f}}(\tau, \boldsymbol{\rho})= & \sum_{\substack{\boldsymbol{m} \in A+\boldsymbol{\mu} \\
B(\boldsymbol{m}+\boldsymbol{b}, J) / B(\boldsymbol{f}, J) \in[0,1)}} \sum_{n \in \mathbb{Z}}(-1)^{B\left(\boldsymbol{m}, K_{\ell}\right)} q^{-\boldsymbol{m}^{2} / 2} e^{-2 \pi i B(\boldsymbol{\rho}, \boldsymbol{m})} \\
\times & \frac{1}{2}(\operatorname{sgn}(B(\boldsymbol{m}+\boldsymbol{b}, J)+n B(\boldsymbol{f}, J))-\operatorname{sgn}(B(\boldsymbol{m}+\boldsymbol{b}, \boldsymbol{f}))) \\
& \times(-1)^{n B\left(\boldsymbol{f}, K_{\ell}\right)} q^{-n B(\boldsymbol{f}, \boldsymbol{m})} e^{-2 \pi i n B(\boldsymbol{\rho}, \boldsymbol{f})} .
\end{aligned}
$$


Carrying out the sum over $n$ as a geometric series, we find

$$
\Theta_{\boldsymbol{\mu}}^{J \boldsymbol{f}}(\tau, \boldsymbol{\rho})=\sum_{\substack{\boldsymbol{m} \in \Lambda+\boldsymbol{\mu} \\ B(\boldsymbol{m}+\boldsymbol{b}, J) / B(\boldsymbol{f}, J) \in[0,1)}} \frac{(-1)^{B\left(\boldsymbol{m}, K_{\ell}\right)} q^{-\boldsymbol{m}^{2} / 2} e^{-2 \pi i B(\boldsymbol{\rho}, \boldsymbol{m})}}{1-q^{-B(\boldsymbol{f}, \boldsymbol{m})} e^{-2 \pi i B(\boldsymbol{\rho}, \boldsymbol{f})}}
$$

where we used that $B\left(\boldsymbol{f}, K_{\ell}\right)=-2$.

Of particular interest in the literature is the suitable polarization $J_{\epsilon}=(\epsilon(\boldsymbol{C}+\ell \boldsymbol{f})+$ $\boldsymbol{f}) / \sqrt{\ell \epsilon^{2}+\epsilon}$, with $\epsilon$ sufficiently small such that no walls are crossed between $\boldsymbol{f}$ and $J_{\epsilon}$ for the rank two vector bundles. If $B(\boldsymbol{\mu}, \boldsymbol{f}) \in \mathbb{Z}+\frac{1}{2}$ the condition on $\boldsymbol{m}$ has no solutions in agreement with the fact there are no stable bundles for such metrics. If $B(\boldsymbol{\mu}, \boldsymbol{f}) \in \mathbb{Z}$ we have the solutions $\boldsymbol{m}=0$ and $\boldsymbol{m}=\frac{1}{2} f$, due to strictly semi-stable bundles. One finds therefore

$$
\begin{aligned}
\Theta_{0}^{J_{\epsilon} \boldsymbol{f}}(\tau, \boldsymbol{\rho}) & =\frac{1}{1-e^{-2 \pi i B(\boldsymbol{\rho}, \boldsymbol{f})}}, \\
\Theta_{\boldsymbol{f}}^{J_{\epsilon} J^{\prime}}(\tau, \boldsymbol{\rho}) & =\frac{-e^{-\pi i B(\boldsymbol{\rho}, \boldsymbol{f})}}{1-e^{-2 \pi i B(\boldsymbol{\rho}, \boldsymbol{f})}}=\frac{i}{2 \sin (\pi B(\boldsymbol{\rho}, \boldsymbol{f}))} .
\end{aligned}
$$

Using (2.7) and letting $\boldsymbol{x}=x_{C} \boldsymbol{C}+x_{f} \boldsymbol{f} \in H^{2}\left(\mathbb{F}_{\ell}, \mathbb{R}\right)$, we arrive at the following nonvanishing generating function for Donaldson invariants for the suitable polarization $J_{\epsilon}$,

$$
\begin{aligned}
\Phi_{0}^{J_{\epsilon}}(p, \boldsymbol{x}) & =-16\left[\left(u^{2}-1\right) \frac{d a}{d u} e^{2 p u+\boldsymbol{x}^{2} G(u)} \cot \left(\frac{1}{2} x_{C} d u / d a\right)\right]_{q^{0}}, \\
\Phi_{\frac{1}{2} \boldsymbol{f}}^{J_{\epsilon}}(p, \boldsymbol{x}) & =16\left[\left(u^{2}-1\right) \frac{d a}{d u} e^{2 p u+\boldsymbol{x}^{2} G(u)} \frac{1}{\sin \left(\frac{1}{2} x_{C} d u / d a\right)}\right]_{q^{0}},
\end{aligned}
$$

where we expressed $\Phi_{0}^{J_{\epsilon}}$ in terms of $\cot (x)$ using the fact that only odd powers of $x_{f}$ contribute to the expansion of the right hand side. This is in agreement with [14, Theorem 5.3] and [2, section 8.2].

\subsection{Application to the projective plane $\mathbb{P}^{2}$}

We consider the complex projective plane $\mathbb{P}^{2}$ as another application of indefinite theta functions to the $u$-plane integral. Since $b_{2}\left(\mathbb{P}^{2}\right)=1$ in this case, the period point of the metric is proportional to the hyperplane class $H$. Since there is thus no chamber dependence, we omit it from the notation. The sum over $\mathrm{U}(1)$ fluxes $\Psi_{\mu}$ is given by ${ }^{7}$

$$
\begin{aligned}
\Psi_{\mu}(\tau, \rho)= & \exp \left(-2 \pi \tau_{2} b^{2}\right) \sum_{k \in \mathbb{Z}+\mu} \partial_{\bar{\tau}}\left(\sqrt{2 \tau_{2}}(k+b)\right) \\
& \times(-1)^{3 k} \bar{q}^{k^{2} / 2} e^{-2 \pi i \bar{\rho} k}
\end{aligned}
$$

where we have used that the canonical class $K_{\mathbb{P}^{2}}$ equals $3 H$.

Since the lattice $H^{2}\left(\mathbb{P}^{2}, \mathbb{Z}\right)$ is one-dimensional, we can not directly apply the indefinite theta function to integrate over the Coulomb branch. However, we can extend the onedimensional lattice to a two-dimensional lattice by dividing and multiplying by the Jacobi

\footnotetext{
${ }^{7}$ We omit the boldface font here for $k, b$ and $\rho$, since they are elements of one-dimensional spaces.
} 
theta function $\theta_{4}$ defined in (A.8): $\Psi_{\mu}=\frac{\theta_{4}(\tau)}{\theta_{4}(\tau)} \Psi_{\mu}$. Geometrically one may interpret these manipulations in terms of the blow-up $\widehat{\mathbb{P}}^{2}$ of $\mathbb{P}^{2}$; note that the measure (3.20) differs by a factor $\theta_{4}^{-1}$ for $\mathbb{P}^{2}$ and $\widehat{\mathbb{P}}^{2}$. Including the summation over $\mathbb{Z}$ in $\theta_{4}$ in the lattice sum, $\Psi_{\mu}(\tau, \rho)$ reads

$$
\begin{aligned}
\Psi_{\mu}(\tau, \rho)= & \frac{\exp \left(-2 \pi \tau_{2} b^{2}\right)}{\theta_{4}(\tau)} \\
& \times \sum_{\left(k_{1}, k_{2}\right) \in \mathbb{Z}^{2}+(\mu, 0)} \partial_{\bar{\tau}}\left(\sqrt{2 \tau_{2}}\left(k_{1}+b\right)\right)(-1)^{3 k_{1}+k_{2}} \bar{q}^{k_{1}^{2} / 2} q^{k_{2}^{2} / 2} e^{-2 \pi i \bar{\rho} k_{1}} .
\end{aligned}
$$

Our earlier discussion shows that $\Psi_{\mu}(\tau, \rho)$ can be expressed as an anti-holomorphic derivative,

$$
\frac{1}{\theta_{4}(\tau)} \partial_{\bar{\tau}} \widehat{\Theta}_{\mu}^{J J^{\prime}}(\tau, \rho)
$$

where $\widehat{\Theta}_{\boldsymbol{\mu}}^{J J^{\prime}}$ is the completion of the indefinite theta function $\Theta_{\boldsymbol{\mu}}^{J J^{\prime}}$ whose associated lattice $\Lambda$ is the two-dimensional lattice with diagonal quadratic form $\operatorname{diag}(1,-1)$. The twodimensional parameters $\boldsymbol{\mu}$ and $\boldsymbol{\rho}$ are given by $(\mu, 0)$ and $(\rho, 0)$ respectively, whereas the two parameters $J, J^{\prime} \in \Lambda \otimes \mathbb{R}$ are given by $J=(1,0)$ and $J^{\prime}=(1,1)$ respectively.

The lattice sum in $\Theta_{\boldsymbol{\mu}}^{J J^{\prime}}$ can be partially carried out using a geometric series, leading to the expression

$$
\Theta_{\mu}^{J J^{\prime}}(\tau, \rho)=w^{\mu}(-1)^{2 \mu} \sum_{\ell \in \mathbb{Z}+\mu} \frac{(-1)^{\ell} q^{\frac{1}{2} \ell^{2}+\mu \ell}}{1-w q^{\ell}},
$$

with $w=e^{2 \pi i \rho}$. This is, up to a prefactor, a specialization of the Appell sum [18]

$$
A(u, v, \tau)=e^{\pi i u} \sum_{n \in \mathbb{Z}} \frac{(-1)^{n} q^{n(n+1) / 2} e^{2 \pi i n v}}{1-e^{2 \pi i u} q^{n}} .
$$

Treating first the case $\mu=\frac{1}{2}$, we arrive at the following expression for the generating function $\Phi_{\frac{1}{2}}(p, x)$

$$
\Phi_{\frac{1}{2}}(p, x)=-32 i\left[\left(u^{2}-1\right) \frac{d a}{d u} e^{2 p u+x^{2} G(u)} \Theta_{\left(\frac{1}{2}, 0\right)}^{J J^{\prime}}(\tau, \rho)\right]_{q^{0}},
$$

which gives for the first few terms

$$
\Phi_{\frac{1}{2}}(0, x)=1+\frac{3}{16} \frac{x^{4}}{4 !}+\frac{29}{32} \frac{x^{8}}{8 !}+\frac{69525}{4096} \frac{x^{12}}{12 !}+O\left(x^{16}\right) .
$$

These terms are in agreement with [17, Theorem 4.4], while the full series matches the expression of Göttsche [13, Theorem 3.5].

Next we consider $\mu=0$. The series $\Phi_{0}(p, x)$ can be determined similarly using multiplication and division by $\theta_{4}$. However, we notice from $(4.21)$ that $\widehat{\Theta}_{0}(\tau, \rho)$ is then divergent for small $\rho$, which is at odds with the Donaldson invariants being polynomials in $\boldsymbol{x}$. The resolution is that the holomorphic integration constant mentioned below (C.4) is non-vanishing in this case. Using the blow-up formula, one finds that the constant equals

$$
C(\tau, \rho)=\frac{\theta_{4}(\tau, 0)}{\theta_{4}(\tau, \rho)} \partial_{\rho} \ln \left(\frac{\theta_{1}(\tau, \rho)}{\theta_{4}(\tau, \rho)}\right)
$$


leading to the following expression for $\Phi_{0}(p, x)$

$$
\Phi_{0}(p, x)=-32 i\left[\left(u^{2}-1\right) \frac{d a}{d u} e^{2 p u+x^{2} G(u)}\left(C(\tau, \rho)+e^{-\pi i \rho} A\left(\rho,-\frac{1}{2} \tau, \tau\right)\right)\right]_{q^{0}} .
$$

To relate this to the expression of [13, Theorem 3.5]. We recall the periodicity property of the Appell function (4.22) from [18, chapter 1],

$$
\frac{A(u, v, \tau)}{\theta_{1}(v, \tau)}-\frac{A(u+z, v+z, \tau)}{\theta_{1}(v+z, \tau)}=\frac{\eta^{3} \theta_{1}(u+v+z, \tau) \theta_{1}(z, \tau)}{\theta_{1}(u, \tau) \theta_{1}(v, \tau) \theta_{1}(u+z, \tau) \theta_{1}(v+z, \tau)} .
$$

Letting $v=-\frac{1}{2} \tau$ and taking the limit $z \rightarrow \frac{1}{2} \tau$, one finds

$$
\begin{aligned}
C(\tau, \rho)+e^{-\pi i \rho} A\left(\rho,-\frac{1}{2} \tau, \tau\right)= & \frac{\theta_{4}(\tau)}{\eta(\tau)^{3}} \sum_{\substack{k_{1} \in \mathbb{Z} \\
k_{2} \in \mathbb{Z}+\frac{1}{2}}}\left(\operatorname{sgn}\left(k_{1}+a\right)-\operatorname{sgn}\left(k_{1}+k_{2}+a\right)\right) \\
& \times k_{2}(-1)^{k_{1}+k_{2}} e^{2 \pi i \rho k_{1}} q^{-k_{1}^{2} / 2+k_{2}^{2} / 2} .
\end{aligned}
$$

Substitution of this expression in (4.25) reproduces the expression in [13, Theorem 3.5]. For completeness, we list the first few terms in the expansion

$$
\Phi_{0}(p, x)=-\frac{3}{2} x+\frac{x^{5}}{5 !}+3 \frac{x^{9}}{9 !}+54 \frac{x^{13}}{13 !}+O\left(x^{17}\right),
$$

in agreement with [17, Theorem 4.2] and [2]. One can arrive at the r.h.s. of (4.27) alternatively by multiplying and dividing in (4.19) by $\theta_{1}(z, \tau)$ rather than $\theta_{4}(\tau)$, and taking the limit $z \rightarrow 0$ in $\Theta_{0}^{J J^{\prime}}(\tau, \rho, z) / \theta_{1}(z, \tau)$. A similar procedure was used in the context of D3-instanton corrections [51, section 4].

\section{On the extension to gauge groups with rank $>1$}

Donaldson-Witten theory can be generalized to theories with a gauge group $G$ with rank $r>1[3,52]$ using the corresponding Seiberg-Witten geometries [53-56]. Mochiziku [57] developed an algebraic-geometric framework to discuss higher rank Donaldson invariants. This section generalizes the $\overline{\mathcal{Q}}$-exact surface operator (2.11) to theories with gauge group $G$, and discusses the sum over $\mathrm{U}(1)^{r}$ fluxes of the Coulomb branch integrand for a fourmanifold $M$ with $b_{1}=0$ and $b_{2}^{+}=1$. We keep this section relatively short and refer the reader for the details to [52].

Let us consider the Coulomb branch of a $\mathcal{N}=2$ supersymmetric Yang-Mills theory whose gauge group $G$ has rank $r$. We denote the Cartan elements of the Lie algebra by $H_{K}, K=1, \ldots, r$. Then, the vacuum expectation value of the scalar component of the $\mathcal{N}=1$ chiral superfield can classicaly be brought to the form

$$
\phi=\sum_{K=1}^{r} a^{K} H_{K} .
$$

The $a^{K}$ provide local special coordinates on the Coulomb branch moduli space. Alternatively, one can consider the $r$ Weyl invariant Casimirs $u_{K}, K=1, \ldots, r$, as coordinates on 
the Coulomb branch. At a generic point on the Coulomb branch, the field content consists of $r$ copies of the effective $\mathrm{U}(1)$ theory described in section 2, which are distinguished by a superscript: $A^{K}, \psi^{K}, \ldots$, for $K=1, \ldots, r$. The effective coupling $\tau_{K L}=\tau_{K L}\left(a^{M}\right)$ is now an $r \times r$ matrix. The effective Coulomb branch theory breaks down at the locus where gauge bosons become massless, or more geometrically, the corresponding Seiberg-Witten curve becomes singular.

Most aspects of the rank one Donaldson-Witten theory generalize to rank $r$ without much effort. For example after topological twisting, the action of the $\overline{\mathcal{Q}}$ operator on the fields is given by

$$
\begin{aligned}
& {\left[\overline{\mathcal{Q}}, A^{K}\right]=\psi^{K}, \quad\left[\overline{\mathcal{Q}}, a^{K}\right]=0, \quad\left[\overline{\mathcal{Q}}, \bar{a}^{K}\right]=\sqrt{2} i \eta^{K},} \\
& {\left[\overline{\mathcal{Q}}, D^{K}\right]=\left(d_{A} \psi^{K}\right)_{+}, \quad\left\{\overline{\mathcal{Q}}, \psi^{K}\right\}=4 \sqrt{2} d a^{K},} \\
& \left\{\overline{\mathcal{Q}}, \eta^{K}\right\}=0, \quad\left\{\overline{\mathcal{Q}}, \chi^{K}\right\}=i\left(F_{+}-D\right)^{K} .
\end{aligned}
$$

The effective Lagrangian on the Coulomb branch is similarly a straightforward generalization of the rank 1 case [52].

There is a larger freedom for the construction of surface operators in the higher rank theories. Starting from any invariant function $\mathcal{U}=\mathcal{U}\left(a^{K}\right)$ of the coordinates $a^{K}$, one may construct a suitable surface operator. The operator $\tilde{I}_{-}(2.10)$ generalized to general $r$ takes the form [52]

$$
\tilde{I}_{-}(\boldsymbol{x})=\int_{\boldsymbol{x}} \frac{i}{32 \sqrt{2} \pi} \mathcal{U}_{K L} \psi^{K} \wedge \psi^{L}-\frac{i}{4 \pi} \mathcal{U}_{K}\left(F_{-}+D\right)^{K},
$$

where the subscripts indicate differentiation to $a^{K}$ :

$$
\mathcal{U}_{K}=\frac{d \mathcal{U}}{d a^{K}}, \quad \mathcal{U}_{K L}=\frac{d^{2} \mathcal{U}}{d a^{K} d a^{L}} .
$$

The generalization of the $\overline{\mathcal{Q}}$-exact surface operator $\tilde{I}_{+}(2.12)$ is similarly given by

$$
\tilde{I}_{+}(\boldsymbol{x})=-\frac{1}{4 \pi} \int_{\boldsymbol{x}}\left\{\overline{\mathcal{Q}}, \overline{\mathcal{U}}_{K} \chi^{K}\right\}
$$

which using the algebra (5.2) becomes

$$
\tilde{I}_{+}(\boldsymbol{x})=-\frac{i}{2 \sqrt{2} \pi} \int_{\boldsymbol{x}} \overline{\mathcal{U}}_{K L} \eta^{K} \chi^{L}+\frac{1}{\sqrt{2}} \overline{\mathcal{U}}_{K}\left(F_{+}-D\right)^{K} .
$$

Our next aim is to derive the sum over the $\mathrm{U}(1)^{r}$ fluxes $\boldsymbol{k}^{K}, \Psi_{r, \mu}^{J}$, when both $\tilde{I}_{+}$and $\tilde{I}_{-}$are inserted in the path integral. After integrating out the auxiliary fields $D^{K}$, we find that $\Psi_{r, \mu}^{J}$ is given by

$$
\begin{aligned}
& \Psi_{r, \mu}^{J}\left(\tau_{K L}, \boldsymbol{\rho}_{K}\right)=\frac{1}{\sqrt{\operatorname{det} v}} e^{-2 \pi v_{K L} \boldsymbol{b}_{+}^{K} \boldsymbol{b}_{+}^{L}} \sum_{\boldsymbol{k} \in \Gamma}(-1)^{B\left(\boldsymbol{k}^{K} W_{K}, K_{M}\right)} \mathcal{K}(\boldsymbol{k}, \boldsymbol{\rho}, \omega) \\
& \times \exp \left(-i \pi \bar{\tau}_{K L} B\left(\boldsymbol{k}_{+}^{K}, \boldsymbol{k}_{+}^{L}\right)-i \pi \tau_{K L} B\left(\boldsymbol{k}_{-}^{K}, \boldsymbol{k}_{-}^{L}\right)-2 \pi i B\left(\boldsymbol{k}_{+}^{K}, \overline{\boldsymbol{\rho}}_{K}\right)-2 \pi i B\left(\boldsymbol{k}_{-}^{K}, \boldsymbol{\rho}_{K}\right)\right)
\end{aligned}
$$

where $v_{K L}=\operatorname{Im}\left(\tau_{K L}\right), W_{K}$ are the components of the Weyl vector of $G$, and we introduced

$$
\boldsymbol{\rho}_{K} \equiv \frac{\boldsymbol{x}}{2 \pi} \mathcal{U}_{K} \in H^{2}(M, \mathbb{C}), \quad \boldsymbol{b}^{K}=v^{K L} \operatorname{Im}\left(\boldsymbol{\rho}_{L}\right) \in H^{2}(M, \mathbb{R})
$$


in analogy to the rank one case. The kernel $\mathcal{K}$ in (5.5) is given by the integral over the fermion zero modes

$$
\begin{array}{r}
\mathcal{K}(\boldsymbol{k}, \boldsymbol{\rho}, \omega)=\int\left[\prod_{K, L=1}^{r} d \eta_{0}^{K} d \chi_{0}^{L}\right] \exp \left(-\frac{\sqrt{2} i}{4} \int_{M} \overline{\mathcal{F}}_{K L M} \eta_{0}^{K} \chi_{0}^{L} \wedge\left(\boldsymbol{k}_{+}-\boldsymbol{b}_{+}\right)^{M}\right. \\
\left.\quad-\frac{i}{\sqrt{2}} \overline{\boldsymbol{\rho}}_{K L} \eta_{0}^{K} \chi_{0}^{L}+\frac{1}{64 \pi} v^{K P} \overline{\mathcal{F}}_{K L M} \overline{\mathcal{F}}_{P Q R} \eta_{0}^{L} \chi_{0}^{M} \wedge \eta_{0}^{Q} \chi_{0}^{R}\right)
\end{array}
$$

where $\mathcal{F}_{K L M}=d \tau_{K L} / d a^{M}$. Carrying out this integral for $G=\mathrm{SU}(3)(r=2)$, we arrive at

$$
\begin{aligned}
\mathcal{K}(\boldsymbol{k}, \boldsymbol{\rho}, \omega)= & \frac{1}{8}\left(\overline{\mathcal{F}}_{11 K} B\left(\boldsymbol{k}^{K}-\boldsymbol{b}^{K}, J\right)+2 B\left(\overline{\boldsymbol{\rho}}_{11}, J\right)\right)\left(\overline{\mathcal{F}}_{22 L} B\left(\boldsymbol{k}^{L}-\boldsymbol{b}^{L}, J\right)+2 B\left(\overline{\boldsymbol{\rho}}_{22}, J\right)\right) \\
& -\frac{1}{8}\left(\overline{\mathcal{F}}_{12 K} B\left(\boldsymbol{k}^{K}-\boldsymbol{b}^{K}, J\right)+2 B\left(\overline{\boldsymbol{\rho}}_{12}, J\right)\right)\left(\overline{\mathcal{F}}_{12 L} B\left(\boldsymbol{k}^{L}-\boldsymbol{b}^{L}, J\right)+2 B\left(\overline{\boldsymbol{\rho}}_{12}, J\right)\right) \\
& +\frac{1}{32 \pi}\left(\overline{\mathcal{F}}_{11 K} \overline{\mathcal{F}}_{22 L}-\overline{\mathcal{F}}_{12 K} \overline{\mathcal{F}}_{12 L}\right) v^{K L} .
\end{aligned}
$$

We leave it for future work to express $\Psi_{r, \mu}^{J}$ as a total derivative of $\bar{a}^{K}$.

\section{Conclusion and discussion}

We have discussed partition functions of Donaldson-Witten theory for a four-manifold $M$ with $b_{2}^{+}=1$, and in particular the contribution of the integral over the Coulomb branch. We have shown that for gauge group $\mathrm{SU}(2)$ and $\mathrm{SO}(3)$ the integrand may be expressed as $\bar{\tau}$-derivative of an indefinite theta function, after insertion of a $\overline{\mathcal{Q}}$-exact surface operator $\tilde{I}_{+}$ which couples to the self-dual part of the field strength $F$. This allows to readily evaluate the integral, and to express it as a sum over the cusps of the integration domain. In this way, we reproduce the result of Göttsche, who expressed generating series of Donaldson invariants in terms of a residue of an indefinite theta function.

There are various directions to which our results may be applied and generalized, in particular the evaluation of partition functions of other four-dimensional theories, such as those including matter and higher rank gauge groups. Besides the fundamental interest in path integrals of Yang-Mills theories, this may also prove useful for establishing new four-manifold invariants [58].

\section{Acknowledgments}

We would like to thank Gregory Moore and Samson Shatashvili for useful discussions and correspondence. JM thanks IHES for hospitality during the completion of this work.

\section{A Modular forms and theta functions}

We collect in this appendix a few essential aspects of the theory of modular forms. For more comprehensive treatments we refer the reader to the available literature. See for example [59-61]. 
Modular groups. The modular group $\mathrm{SL}(2, \mathbb{Z})$ is the group of integer matrices with unit determinant

$$
\mathrm{SL}(2, \mathbb{Z})=\left\{\left(\begin{array}{ll}
a & b \\
c & d
\end{array}\right) \mid a, b, c, d \in \mathbb{Z} ; a d-b c=1\right\} .
$$

We introduce moreover the congruence subgroup $\Gamma^{0}(n)$

$$
\Gamma^{0}(n)=\left\{\left(\begin{array}{ll}
a & b \\
c & d
\end{array}\right) \in \mathrm{SL}(2, \mathbb{Z}) \mid b=0 \bmod n\right\} .
$$

Eisenstein series. We let $\tau \in \mathbb{H}$ and define $q=e^{2 \pi i \tau}$. Then the Eisenstein series $E_{k}: \mathbb{H} \rightarrow \mathbb{C}$ for even $k \geq 2$ are defined as the $q$-series

$$
E_{k}(\tau)=1-\frac{2 k}{B_{k}} \sum_{n=1}^{\infty} \sigma_{k-1}(n) q^{n}
$$

with $\sigma_{k}(n)=\sum_{d \mid n} d^{k}$ the divisor sum. For $k \geq 4, E_{k}$ is a modular form of $\operatorname{SL}(2, \mathbb{Z})$ of weight $k$. In other words, it transforms under $\operatorname{SL}(2, \mathbb{Z})$ as

$$
E_{k}\left(\frac{a \tau+b}{c \tau+d}\right)=(c \tau+d)^{k} E_{k}(\tau)
$$

On the other hand $E_{2}$ is a quasi-modular form, which means that the $\operatorname{SL}(2, \mathbb{Z})$ transformation of $E_{2}$ includes a shift in addition to the weight,

$$
E_{2}\left(\frac{a \tau+b}{c \tau+d}\right)=(c \tau+d)^{2} E_{2}(\tau)-\frac{6 i}{\pi} c(c \tau+d) .
$$

Dedekind eta function. The Dedekind eta function $\eta: \mathbb{H} \rightarrow \mathbb{C}$ is defined as

$$
\eta(\tau)=q^{\frac{1}{24}} \prod_{n=1}^{\infty}\left(1-q^{n}\right)
$$

It is a modular form of weight $\frac{1}{2}$ under $\mathrm{SL}(2, \mathbb{Z})$ with a non-trivial multiplier system. It transforms under the generators of $\mathrm{SL}(2, \mathbb{Z})$ as

$$
\begin{aligned}
& \eta(-1 / \tau)=-i \sqrt{-i \tau} \eta(\tau), \\
& \eta(\tau+1)=e^{\frac{\pi i}{12}} \eta(\tau) .
\end{aligned}
$$

Jacobi theta functions. The four Jacobi theta functions $\vartheta_{j}: \mathbb{H} \times \mathbb{C} \rightarrow \mathbb{C}, j=1, \ldots, 4$, are defined as

$$
\begin{aligned}
& \vartheta_{1}(\tau, v)=i \sum_{r \in \mathbb{Z}+\frac{1}{2}}(-1)^{r-\frac{1}{2}} q^{r^{2} / 2} e^{2 \pi i r v} \\
& \vartheta_{2}(\tau, v)=\sum_{r \in \mathbb{Z}+\frac{1}{2}} q^{r^{2} / 2} e^{2 \pi i r v} \\
& \vartheta_{3}(\tau, v)=\sum_{n \in \mathbb{Z}} q^{n^{2} / 2} e^{2 \pi i n v} \\
& \vartheta_{4}(\tau, v)=\sum_{n \in \mathbb{Z}}(-1)^{n} q^{n^{2} / 2} e^{2 \pi i n v}
\end{aligned}
$$


We let $\vartheta_{j}(\tau, 0)=\vartheta_{j}(\tau)$ for $j=2,3,4$. Their transformations under the generators of $\Gamma^{0}(4)$ are

$$
\begin{array}{ll}
\vartheta_{2}(\tau+4)=-\vartheta_{2}(\tau), & \vartheta_{2}\left(\frac{\tau}{\tau+1}\right)=\sqrt{\tau+1} \vartheta_{3}(\tau), \\
\vartheta_{3}(\tau+4)=\vartheta_{3}(\tau), & \vartheta_{3}\left(\frac{\tau}{\tau+1}\right)=\sqrt{\tau+1} \vartheta_{2}(\tau), \\
\vartheta_{4}(\tau+4)=\vartheta_{4}(\tau), & \vartheta_{4}\left(\frac{\tau}{\tau+1}\right)=e^{-\frac{\pi i}{4} \sqrt{\tau+1}} \vartheta_{4}(\tau) .
\end{array}
$$

Siegel-Narain theta function. Siegel-Narain theta functions form a large class of theta functions of which the Jacobi theta functions are a special case. We restrict here to a specific Siegel-Narain theta function for which the associated lattice $\Lambda$ is a uni-modular lattice of signature $(1, n-1)$ (or a Lorentzian lattice). We denote the bilinear form by $B(\boldsymbol{x}, \boldsymbol{y})$ and the quadratic form $B(\boldsymbol{x}, \boldsymbol{x}) \equiv Q(\boldsymbol{x}) \equiv \boldsymbol{x}^{2}$. Let $K$ be a characteristic vector of $\Lambda$, such that $Q(\boldsymbol{k})+B(\boldsymbol{k}, K) \in 2 \mathbb{Z}$ for each $\boldsymbol{k} \in \Lambda$.

Given an element $J \in \Lambda \otimes \mathbb{R}$ with $Q(J)>0$, we may decompose the space $\Lambda \otimes \mathbb{R}$ in a positive definite subspace $\Lambda_{+}$spanned by $J$, and a negative definite subspace $\Lambda_{-}$, orthogonal to $\Lambda_{+}$. Let $\underline{J}=J / \sqrt{Q(J)}$ be the normalization of $J$. The projections of a vector $\boldsymbol{k} \in \Lambda$ to $\Lambda_{+}$and $\Lambda_{-}$are then given by

$$
\boldsymbol{k}_{+}=B(\boldsymbol{k}, \underline{J}) \underline{J}, \quad \boldsymbol{k}_{-}=\boldsymbol{k}-\boldsymbol{k}_{+}
$$

Given this notation, we can introduce the Siegel-Narain theta of our interest $\Psi_{\mu}^{J}$ : $\mathbb{H} \times \mathbb{C} \rightarrow \mathbb{C}$. Let $J$ be as discussed above $\left(\right.$ A.10) and $\boldsymbol{\mu} \in \Lambda \otimes \mathbb{R}$. Then $\Psi_{\mu}^{J}$ is defined by ${ }^{8}$

$$
\begin{aligned}
\Psi_{\boldsymbol{\mu}}^{J}(\tau, \boldsymbol{z})= & e^{-2 \pi \tau_{2} \boldsymbol{b}_{+}^{2}} \sum_{\boldsymbol{k} \in \Lambda+\boldsymbol{\mu}} \partial_{\bar{\tau}}\left(\sqrt{2 \tau_{2}} B(\boldsymbol{k}+\boldsymbol{b}, \underline{J})\right)(-1)^{B(\boldsymbol{k}, K)} q^{-\boldsymbol{k}_{-}^{2} / 2} \bar{q}^{2} / 2 \\
& \times e^{-2 \pi i B\left(\boldsymbol{z}, \boldsymbol{k}_{-}\right)-2 \pi i B\left(\overline{\boldsymbol{z}}, \boldsymbol{k}_{+}\right)}
\end{aligned}
$$

where $\boldsymbol{b}=\operatorname{Im}(\boldsymbol{z}) / \tau_{2} \in \Lambda \otimes \mathbb{R}$. The parameter $\boldsymbol{b}$ is typically taken independent of $\bar{\tau}$ in the literature, and in that case (A.11) simplifies. In the application in the main text, $\boldsymbol{z}$ is actually a modular form of weight -1 , such that $\boldsymbol{b}$ is not independent of $\bar{\tau}$. The derivative $\partial_{\bar{\tau}} \boldsymbol{b}$ transforms then as a modular form of mixed weight $(1,2)$.

To determine the modular properties of $\Psi_{\mu}^{J}$, one may use the standard technique of Poisson resummation, as for example in [22]. To this end, it is most convenient to shift $\boldsymbol{\mu}$ by $K / 2$. One finds for the modular transformations of $\Psi_{\boldsymbol{\mu}}^{J}$ under the generators of $\operatorname{SL}(2, \mathbb{Z})$ the following identities

$$
\begin{aligned}
\Psi_{\boldsymbol{\mu}+K / 2}^{J}(\tau+1, \boldsymbol{z})= & e^{\pi i\left(\boldsymbol{\mu}^{2}-K^{2} / 4\right)} \Psi_{\boldsymbol{\mu}+K / 2}^{J}(\tau, \boldsymbol{z}+\boldsymbol{\mu}) \\
\Psi_{\boldsymbol{\mu}+K / 2}^{J}(-1 / \tau, \boldsymbol{z} / \tau)= & -i(-i \tau)^{\frac{n}{2}}(i \bar{\tau})^{2} \exp \left(-\pi i \boldsymbol{z}^{2} / \tau+\pi i K^{2} / 2\right)(-1)^{B(\boldsymbol{\mu}, K)} \\
& \times \Psi_{K / 2}^{J}(\tau, \boldsymbol{z}-\boldsymbol{\mu}) .
\end{aligned}
$$

\footnotetext{
${ }^{8}$ For brevity we list in $\Psi_{\mu}^{J}$ only the holomorphic arguments $\tau$ and $z$, even though the function does also depend on $\bar{\tau}$ and $\bar{z}$.
} 
We note that a shift in $\boldsymbol{z}$ by $\boldsymbol{\nu} \in \Lambda \otimes \mathbb{R}$ times $\tau$, can be related to a shift in $\boldsymbol{\mu}$ by the following identity

$$
\Psi_{\boldsymbol{\mu}}^{J}(\tau, \boldsymbol{z}+\boldsymbol{\nu} \tau)=e^{2 \pi i B(\boldsymbol{z}, \boldsymbol{\nu})} q^{\boldsymbol{\nu}^{2} / 2}(-1)^{-B(\boldsymbol{\nu}, \boldsymbol{\mu})} \Psi_{\boldsymbol{\mu}+\boldsymbol{\nu}}^{J}(\tau, \boldsymbol{z})
$$

while shifting $\boldsymbol{z}$ by $\boldsymbol{\nu} \in \Lambda \otimes \mathbb{R}$ gives

$$
\Psi_{\boldsymbol{\mu}}^{J}(\tau, \boldsymbol{z}+\boldsymbol{\nu})=e^{-2 \pi i B(\boldsymbol{\nu}, \boldsymbol{\mu})} \Psi_{\boldsymbol{\mu}}^{J}(\tau, \boldsymbol{z}) .
$$

Due to the relation (A.13), the parameters $\boldsymbol{\mu}$ and $\boldsymbol{z}$ are somewhat redundant as arguments of $\Psi_{\mu}^{J}$. They play however different roles in the main part of this article. There $\boldsymbol{\mu}$ is one half the second Stiefel-Whitney class of a line bundle and therefore restricted to $\Lambda / 2$, while $\boldsymbol{z}$ is a fugacity valued in $\Lambda \otimes \mathbb{C}$. For such $\boldsymbol{\mu} \in \Lambda / 2$ one can show that $\Psi_{\mu}^{J}$ is a modular form of the congruence subgroup $\Gamma^{0}(4)$. The action of the generators of $\Gamma^{0}(4)$ on $\Psi_{\boldsymbol{\mu}}^{J}$ with $\boldsymbol{\mu} \in \Lambda / 2$ is given by

$$
\begin{aligned}
\Psi_{\boldsymbol{\mu}}^{J}(\tau,-\boldsymbol{z}) & =-e^{2 \pi i B(\boldsymbol{\mu}, K)} \Psi_{\boldsymbol{\mu}}^{J}(\tau, \boldsymbol{z}), \\
\Psi_{\boldsymbol{\mu}}^{J}\left(\frac{\tau}{\tau+1}, \frac{\boldsymbol{z}}{\tau+1}\right) & =(\tau+1)^{\frac{n}{2}}(\bar{\tau}+1)^{2} \exp \left(-\frac{\pi i \boldsymbol{z}^{2}}{\tau+1}+\frac{\pi i}{4} K^{2}\right) \Psi_{\boldsymbol{\mu}}^{J}(\tau, \boldsymbol{z}), \\
\Psi_{\boldsymbol{\mu}}^{J}(\tau+4, \boldsymbol{z}) & =e^{2 \pi i B(\boldsymbol{\mu}, K)} \Psi_{\boldsymbol{\mu}}(\tau, \boldsymbol{z}) .
\end{aligned}
$$

\section{B Indefinite theta functions for uni-modular lattices of signature $(1, n-$} 1)

We discuss in this appendix various aspects of indefinite theta functions and their modular completion. We assume that the associated lattice $\Lambda$ is unimodular and of signature $(1, n-$ 1) and use the notation discussed in appendix A.

To define the indefinite theta function, we choose two positive definite vectors $J$ and $J^{\prime} \in \Lambda \otimes \mathbb{R}$ with $B\left(J, J^{\prime}\right)>0$, such that they both lie in the same positive cone of $\Lambda$. Let $\underline{J}$ and $\underline{J}^{\prime}$ be their normalizations. The arguments of theta function are $\tau \in \mathbb{H}, \boldsymbol{z} \in \Lambda \otimes \mathbb{C}$ and $\boldsymbol{\mu} \in \Lambda \otimes \mathbb{R}$. We let $\boldsymbol{b}=\operatorname{Im}(\boldsymbol{z}) / \tau_{2} \in \Lambda \otimes \mathbb{R}$. In terms of this data, the indefinite theta function $\Theta_{\boldsymbol{\mu}}^{J J^{\prime}}$ is defined as

$$
\begin{gathered}
\Theta_{\boldsymbol{\mu}}^{J J^{\prime}}(\tau, \boldsymbol{z})=\sum_{\boldsymbol{k} \in \Lambda+\boldsymbol{\mu}} \frac{1}{2}\left(\operatorname{sgn}(B(\boldsymbol{k}+\boldsymbol{b}, J))-\operatorname{sgn}\left(B\left(\boldsymbol{k}+\boldsymbol{b}, J^{\prime}\right)\right)\right) \\
\times(-1)^{B(\boldsymbol{k}, K)} q^{-\boldsymbol{k}^{2} / 2} e^{-2 \pi i B(\boldsymbol{z}, \boldsymbol{k})}
\end{gathered}
$$

One may show that the sum over $\Lambda$ is convergent [18]. However, $\Theta_{\mu}^{J J^{\prime}}$ does only transform as a modular form after addition of certain non-holomorphic terms. References $[18,19]$ explain that the modular completion $\widehat{\Theta}_{\mu}^{J J^{\prime}}$ of $\Theta_{\mu}^{J J^{\prime}}$ is obtained by substituting (rescaled) error functions for the sgn-functions in (B.1). The completion $\widehat{\Theta}_{\boldsymbol{\mu}}^{J J^{\prime}}$ then transforms as a modular form of weight $n / 2$, and is explicitly given by

$$
\begin{gathered}
\widehat{\Theta}_{\boldsymbol{\mu}}^{J J^{\prime}}(\tau, \boldsymbol{z})=\sum_{\boldsymbol{k} \in \Lambda+\mu} \frac{1}{2}\left(E\left(\sqrt{2 \tau_{2}} B(\boldsymbol{k}+\boldsymbol{b}, \underline{J})\right)-E\left(\sqrt{2 \tau_{2}} B\left(\boldsymbol{k}+\boldsymbol{b}, \underline{J}^{\prime}\right)\right)\right) \\
\times(-1)^{B(\boldsymbol{k}, K)} q^{-\boldsymbol{k}^{2} / 2} e^{-2 \pi i B(\boldsymbol{z}, \boldsymbol{k})}
\end{gathered}
$$


where $E(u): \mathbb{R} \rightarrow[-1,1]$ is a reparametrization of the error function,

$$
E(u)=2 \int_{0}^{u} e^{-\pi t^{2}} d t=\operatorname{Erf}(\sqrt{\pi} u)
$$

Note that in the limit $\tau_{2} \rightarrow \infty, E$ in (B.2) approaches the original sgn-function of (B.1),

$$
\lim _{\tau_{2} \rightarrow \infty} E\left(\sqrt{2 \tau_{2}} u\right)=\operatorname{sgn}(u)
$$

If we analytically continue $E$ to a function with complex argument, then this limit is only convergent for $-\frac{\pi}{4}<\operatorname{Arg}(u)<\frac{\pi}{4}$.

The transformation properties under $\mathrm{SL}(2, \mathbb{Z})$ follow from chapter 2 of Zwegers' thesis [18] or Vignéras [62]. One finds for the action of the generators on $\widehat{\Theta}_{\boldsymbol{\mu}+K / 2}^{J J^{\prime}}(\tau, \boldsymbol{z})$

$$
\begin{aligned}
\widehat{\Theta}_{\boldsymbol{\mu}+K / 2}^{J J^{\prime}}(\tau+1, \boldsymbol{z}) & =e^{\pi i\left(\boldsymbol{\mu}^{2}-K^{2} / 4\right)} \widehat{\Theta}_{\boldsymbol{\mu + K / 2}}^{J J^{\prime}}(\tau, \boldsymbol{z}+\boldsymbol{\mu}), \\
\widehat{\Theta}_{\boldsymbol{\mu}+K / 2}^{J J^{\prime}}(-1 / \tau, \boldsymbol{z} / \tau) & =i(-i \tau)^{n / 2} \exp \left(-\pi i \boldsymbol{z}^{2} / \tau+\pi i K^{2} / 2\right) \widehat{\Theta}_{K / 2}^{J J^{\prime}}(\tau, \boldsymbol{z}-\boldsymbol{\mu}) .
\end{aligned}
$$

For our application, the $\bar{\tau}$-derivative of $\widehat{\Theta}_{\mu}^{J J^{\prime}}$ is of particular interest. This gives the "shadow" 9 of $\Theta_{\boldsymbol{\mu}}^{J J^{\prime}}$, whose modular properties are easier to determine than those of $\Theta_{\boldsymbol{\mu}}^{J J^{\prime}}$. We obtain here

$$
\partial_{\bar{\tau}} \widehat{\Theta}_{\boldsymbol{\mu}}^{J J^{\prime}}(\tau, \boldsymbol{z})=\Psi_{\boldsymbol{\mu}}^{J}(\tau, \boldsymbol{z})-\Psi_{\boldsymbol{\mu}}^{J^{\prime}}(\tau, \boldsymbol{z}),
$$

with $\Psi_{\boldsymbol{\mu}}^{J}$ (A.11) the same function discussed in appendix A. The modular properties of $\Psi_{\boldsymbol{\mu}}^{J}$ are given in (A.12), and can be obtained using standard Poisson resummation.

The completion (B.2) may simplify if the lattice $\Lambda$ contains vectors $\boldsymbol{k}_{0} \in \Lambda$ with norm $\boldsymbol{k}_{0}^{2}=0$. For such lattices $J$ and/or $J^{\prime}$ can be chosen to equal such a vector, and careful analysis of the limit shows that the error function reduces to the original sgn-function [18]. We assume now that $J^{\prime} \in \Lambda$ such that $\left(J^{\prime}\right)^{2}=0$. To ensure convergence of the sum, one needs to require furthermore that $B\left(\boldsymbol{k}+\boldsymbol{b}, J^{\prime}\right) \neq 0$ for any $\boldsymbol{k} \in \Lambda+K / 2+\boldsymbol{\mu}$, except if one also has $B(\boldsymbol{k}+\boldsymbol{b}, J)=0$. Then the completion $\widehat{\Theta}_{\boldsymbol{\mu}}^{J J^{\prime}}$ is given by

$$
\begin{gathered}
\widehat{\Theta}_{\boldsymbol{\mu}}^{J J^{\prime}}(\tau, \boldsymbol{z})=\sum_{\boldsymbol{k} \in \Lambda+K / 2+\mu} \frac{1}{2}\left(E\left(\sqrt{2 \tau_{2}} B(\boldsymbol{k}+\boldsymbol{b}, \underline{J})\right)-\operatorname{sgn}\left(B\left(\boldsymbol{k}+\boldsymbol{b}, J^{\prime}\right)\right)\right) \\
\times(-1)^{B(\boldsymbol{k}, K)} q^{-\boldsymbol{k}^{2} / 2} e^{-2 \pi i B(\boldsymbol{z}, \boldsymbol{k})}
\end{gathered}
$$

with shadow

$$
\partial_{\bar{\tau}} \Theta_{\boldsymbol{\mu}}^{J J^{\prime}}(\tau, \boldsymbol{z})=\Psi_{\boldsymbol{\mu}}^{J}(\tau, \boldsymbol{z}) .
$$

We note that it is important here that $J^{\prime} \in \Lambda$, since $\widehat{\Theta}_{\boldsymbol{\mu}}^{J J^{\prime}}$ is otherwise not convergent. A divergent example is discussed in [51, appendix B.3].

\footnotetext{
${ }^{9}$ Since indefinite theta functions are mixed mock modular forms in general, the notion of "shadow" used here is slightly different from its definition for mock modular forms [19].
} 


\section{Integrating over the fundamental domain}

In this appendix we discuss the recipe we use to evaluate the integral over the $u$-plane. Let $\mathcal{F}_{Y}$ be the compact set, whose boundaries are given by the following arcs

$$
\begin{aligned}
& 1: \quad \tau=\frac{1}{2}+i \tau_{2}, \quad \tau_{2} \in\left[\frac{1}{2} \sqrt{3}, Y\right], \\
& 2: \quad \tau=\tau_{1}+i Y, \quad \tau_{1} \in\left[-\frac{1}{2}, \frac{1}{2}\right], \\
& 3: \quad \tau=-\frac{1}{2}+i \tau_{2}, \quad \tau_{2} \in\left[\frac{1}{2} \sqrt{3}, Y\right], \\
& 4: \quad \tau=i e^{i \varphi}, \quad \varphi \in\left[-\frac{\pi}{6}, \frac{\pi}{6}\right] .
\end{aligned}
$$

We denote the non-compact subset obtained in the limit, $\lim _{Y \rightarrow \infty} \mathcal{F}_{Y}$, by $\mathcal{F}_{\infty}$, which can be chosen as the fundamental domain $\mathcal{F}=\mathbb{H} / \mathrm{SL}(2, \mathbb{Z})$ of the modular group. We are interested in integrals of the form

$$
I_{F}=\lim _{Y \rightarrow \infty} \int_{\mathcal{F}_{Y}} d \tau \wedge d \bar{\tau} F
$$

where $F=F(\tau, \bar{\tau})$ is a non-holomorphic function of $\tau$, which transforms under $\operatorname{SL}(2, \mathbb{Z})$ as

$$
F\left(\frac{a \tau+b}{c \tau+d}, \frac{a \bar{\tau}+b}{c \bar{\tau}+d}\right)=|c \tau+d|^{4} F(\tau, \bar{\tau}),
$$

such that the integrand is modular invariant. Furthermore, we allow that $F$ has a pole at $i \infty$ and its images in $\mathbb{Q}$ under $\mathrm{SL}(2, \mathbb{Z})$, but is regular elsewhere. We now make the crucial assumption that $F$ can be expressed as a total derivative

$$
F=\frac{\partial H}{\partial \bar{\tau}}
$$

where $H=H(\tau, \bar{\tau})$ is a function which transforms as a weight two modular form. Note that $H$ is not unique since adding a weakly holomorphic modular of weight 2 to $H$ does not change (C.4). Assuming that this ambiguity is fixed by other means, we see that the integrand is exact

$$
-d(H d \tau)
$$

Therefore, by Stokes theorem $I_{F}$ equals

$$
I_{F}=-\lim _{Y \rightarrow \infty} \int_{\partial \mathcal{F}_{Y}} H d \tau
$$

Since the integrand is invariant under $\tau \rightarrow \tau+1$, the contribution of the arcs (1) and (3) in (C.1) add up to 0. Moreover, since the integrand is invariant under $\tau \rightarrow-\frac{1}{\tau}$, the contribution due to arc (4) vanishes. Thus what remains is arc (2), which is traversed in counterclockwise direction. As a result we find

$$
I_{F}=-\lim _{Y \rightarrow \infty} \int_{\frac{1}{2}+i Y}^{-\frac{1}{2}+i Y} H d \tau=[H]_{q^{0}},
$$

where the $[H]_{q^{0}}$ denotes the coefficient of $q^{0}$ of $H$. 
Open Access. This article is distributed under the terms of the Creative Commons Attribution License (CC-BY 4.0), which permits any use, distribution and reproduction in any medium, provided the original author(s) and source are credited.

\section{References}

[1] C. Vafa and E. Witten, A strong coupling test of S duality, Nucl. Phys. B 431 (1994) 3 [hep-th/9408074] [INSPIRE].

[2] G.W. Moore and E. Witten, Integration over the u plane in Donaldson theory, Adv. Theor. Math. Phys. 1 (1997) 298 [hep-th/9709193] [INSPIRE].

[3] A. Losev, N. Nekrasov and S.L. Shatashvili, Issues in topological gauge theory, Nucl. Phys. B 534 (1998) 549 [hep-th/9711108] [INSPIRE].

[4] N.A. Nekrasov, Seiberg-Witten prepotential from instanton counting, Adv. Theor. Math. Phys. 7 (2003) 831 [hep-th/0206161] [INSPIRE].

[5] V. Pestun, Localization of gauge theory on a four-sphere and supersymmetric Wilson loops, Commun. Math. Phys. 313 (2012) 71 [arXiv:0712.2824] [INSPIRE].

[6] A. Kapustin, B. Willett and I. Yaakov, Exact results for Wilson loops in superconformal Chern-Simons theories with matter, JHEP 03 (2010) 089 [arXiv:0909.4559] [INSPIRE].

[7] M. Bershtein, G. Bonelli, M. Ronzani and A. Tanzini, Exact results for $N=2$ supersymmetric gauge theories on compact toric manifolds and equivariant Donaldson invariants, JHEP 07 (2016) 023 [arXiv: 1509.00267] [INSPIRE].

[8] E. Witten, Topological quantum field theory, Commun. Math. Phys. 117 (1988) 353 [INSPIRE].

[9] S.K. Donaldson, Polynomial invariants for smooth manifolds, Topology 29 (1990) 257 [INSPIRE].

[10] S.K. Donaldson and P.B. Kronheimer, The geometry of four-manifolds, Clarendon Press, Oxford University Press, Oxford U.K., (1990).

[11] E. Witten, Monopoles and four manifolds, Math. Res. Lett. 1 (1994) 769 [hep-th/9411102] [INSPIRE].

[12] E. Witten, On S duality in Abelian gauge theory, Selecta Math. 1 (1995) 383 [hep-th/9505186] [INSPIRE].

[13] L. Göttsche, Modular forms and Donaldson invariants for 4-manifolds with $b_{2}^{+}=1$, J. Amer. Math. Soc. 9 (1996) 827 [alg-geom/9506018].

[14] L. Göttsche and D. Zagier, Jacobi forms and the structure of Donaldson invariants for 4-manifolds with $b_{2}^{+}=1$, Selecta Math. 4 (1998) 69 [alg-geom/9612020].

[15] W.P. Li and Z. Qin, Lower-degree Donaldson polynomials of rational surfaces, J. Alg. Geom. 2 (1993) 413.

[16] D. Kotschick and P. Lisca, Instanton invariants of $P^{2}$ via topology, Math. Ann. 303 (1995) 345.

[17] G. Ellingsrud and L. Göttsche, Wall-crossing formulas, Bott residue formula and the Donaldson invariants of rational surfaces, Quart. J. Math. Oxford Ser. 49 (1998) 307 [alg-geom/9506019]. 
[18] S.P. Zwegers, Mock theta functions, Ph.D. thesis, Utrecht University, Utrecht The Netherlands, (2008) [arXiv:0807.4834] [INSPIRE].

[19] D. Zagier, Ramanujan's mock theta functions and their applications (after Zwegers and Ono-Bringmann), Astérisque (2009) 143.

[20] L.J. Dixon, V. Kaplunovsky and J. Louis, Moduli dependence of string loop corrections to gauge coupling constants, Nucl. Phys. B 355 (1991) 649 [INSPIRE].

[21] J.A. Harvey and G.W. Moore, Algebras, BPS states and strings, Nucl. Phys. B 463 (1996) 315 [hep-th/9510182] [INSPIRE].

[22] R.E. Borcherds, Automorphic forms with singularities on Grassmannians, Invent. Math. 132 (1998) 491 [alg-geom/9609022] [INSPIRE].

[23] D. Zagier, Nombres de classes et formes modulaires de poids 3/2 (in French), C. R. Acad. Sci. Paris 281 (1975) 883.

[24] A. Malmendier and K. Ono, SO(3)-Donaldson invariants of $C P^{2}$ and mock theta functions, Geom. Topol. 16 (2012) 1767 [arXiv:0808.1442] [InSPIRE].

[25] A. Malmendier, Donaldson invariants of $C P^{1} \times C P^{1}$ and mock theta functions, Commun. Num. Theor. Phys. 5 (2011) 203 [arXiv:1008.0175] [InSPIRE].

[26] M. Griffin, A. Malmendier and K. Ono, SU(2)-Donaldson invariants of the complex projective plane, Forum Math. 27 (2015) 2003 [arXiv:1209.2743] [INSPIRE].

[27] D. Gaiotto, $N=2$ dualities, JHEP 08 (2012) 034 [arXiv:0904.2715] [INSPIRE].

[28] D. Gaiotto, G.W. Moore and A. Neitzke, Wall-crossing, Hitchin systems and the WKB approximation, arXiv:0907.3987 [INSPIRE].

[29] J. Manschot and G.W. Moore, A modern farey tail, Commun. Num. Theor. Phys. 4 (2010) 103 [arXiv:0712.0573] [INSPIRE].

[30] J. Manschot, Stability and duality in $N=2$ supergravity, Commun. Math. Phys. 299 (2010) 651 [arXiv:0906.1767] [INSPIRE].

[31] A. Dabholkar, S. Murthy and D. Zagier, Quantum black holes, wall crossing and mock modular forms, arXiv:1208.4074 [INSPIRE].

[32] S. Alexandrov, S. Banerjee, J. Manschot and B. Pioline, Multiple D3-instantons and mock modular forms I, Commun. Math. Phys. 353 (2017) 379 [arXiv: 1605.05945] [INSPIRE].

[33] M.C.N. Cheng and J.F.R. Duncan, On Rademacher sums, the largest Mathieu group and the holographic modularity of moonshine, Commun. Num. Theor. Phys. 6 (2012) 697 [arXiv:1110.3859] [INSPIRE].

[34] N. Seiberg and E. Witten, Electric-magnetic duality, monopole condensation and confinement in $N=2$ supersymmetric Yang-Mills theory, Nucl. Phys. B 426 (1994) 19 [Erratum ibid. B 430 (1994) 485] [hep-th/9407087] [INSPIRE].

[35] N. Seiberg and E. Witten, Monopoles, duality and chiral symmetry breaking in $N=2$ supersymmetric QCD, Nucl. Phys. B 431 (1994) 484 [hep-th/9408099] [INSPIRE].

[36] L. Álvarez-Gaumé and S.F. Hassan, Introduction to $S$ duality in $N=2$ supersymmetric gauge theories: a pedagogical review of the work of Seiberg and Witten, Fortsch. Phys. 45 (1997) 159 [hep-th/9701069] [INSPIRE]. 
[37] A. Bilal, Duality in $N=2$ SUSY SU(2) Yang-Mills theory: a pedagogical introduction to the work of Seiberg and Witten, in Quantum fields and quantum space time. Proceedings, NATO Advanced Study Institute, Cargese France, 22 July-3 August 1996, pg. 21 [hep-th/9601007] [INSPIRE].

[38] Y.M. Shnir, Magnetic monopoles, Springer-Verlag, Berlin Heidelberg Germany, (2005).

[39] J. Terning, Modern supersymmetry: dynamics and duality, Oxford Science Publications, Oxford U.K., (2009).

[40] Y. Tachikawa, $N=2$ supersymmetric dynamics for pedestrians, Lect. Notes Phys. 890 (2014) 1 [arXiv:1312.2684].

[41] D. Gaiotto, Families of $N=2$ field theories, in New dualities of supersymmetric gauge theories, J. Teschner ed., Springer, Cham Switzerland, (2016), pg. 31 [arXiv:1412.7118].

[42] H. Nakajima and K. Yoshioka, Lectures on instanton counting, in CRM workshop on algebraic structures and moduli spaces, Montreal Canada, 14-20 July 2003 [math/0311058] [INSPIRE].

[43] M. Matone, Instantons and recursion relations in $N=2$ SUSY gauge theory, Phys. Lett. B 357 (1995) 342 [hep-th/9506102] [INSPIRE].

[44] S. Wu, The geometry and physics of the Seiberg-Witten equations, in Geometric analysis and applications to quantum field theory, P. Bouwknegt and S. Wu eds., Birkhäuser, Boston U.S.A., (2002), pg. 157.

[45] G. Korpas, J. Manschot, G.W. Moore and I. Nidaev, to appear.

[46] J. Labastida and M. Marino, Topological quantum field theory and four manifolds, Springer, Dordrecht The Netherlands, (2005).

[47] E.P. Verlinde, Global aspects of electric-magnetic duality, Nucl. Phys. B 455 (1995) 211 [hep-th/9506011] [INSPIRE].

[48] H. Petersson, Konstruktion der Modulformen und der zu gewissen Grenzkreisgruppen gehörigen automorphen Formen von positiver reeller Dimension und die vollständige Bestimmung ihrer Fourierkoeffizienten (in German), S. B. Heidelberger Akad. Wiss. Math. Nat. Kl. (1950) 417.

[49] W. Lerche, A.N. Schellekens and N.P. Warner, Lattices and strings, Phys. Rept. 177 (1989) 1 [INSPIRE].

[50] W.P. Barth, K. Hulek, C.A.M. Peters and A. Van de Ven, Compact complex surfaces, 2 ed., Springer-Verlag, Berlin Heidelberg German, (2004).

[51] S. Alexandrov, S. Banerjee, J. Manschot and B. Pioline, Multiple D3-instantons and mock modular forms II, arXiv: 1702.05497 [INSPIRE].

[52] M. Mariño and G.W. Moore, The Donaldson-Witten function for gauge groups of rank larger than one, Commun. Math. Phys. 199 (1998) 25 [hep-th/9802185] [InSPIRE].

[53] A. Klemm, W. Lerche, S. Yankielowicz and S. Theisen, Simple singularities and $N=2$ supersymmetric Yang-Mills theory, Phys. Lett. B 344 (1995) 169 [hep-th/9411048] [INSPIRE].

[54] P.C. Argyres and A.E. Faraggi, The vacuum structure and spectrum of $N=2$ supersymmetric $\mathrm{SU}(N)$ gauge theory, Phys. Rev. Lett. 74 (1995) 3931 [hep-th/9411057] [INSPIRE]. 
[55] M.R. Douglas and S.H. Shenker, Dynamics of SU(N) supersymmetric gauge theory, Nucl. Phys. B 447 (1995) 271 [hep-th/9503163] [InSPIRE].

[56] Y. Tachikawa and S. Terashima, Seiberg-Witten geometries revisited, JHEP 09 (2011) 010 [arXiv:1108.2315] [INSPIRE].

[57] T. Mochiziku, Donaldson type invariants for algebraic surfaces, Lect. Notes Math. 1972 (2009) 1.

[58] M. Mariño, G.W. Moore and G. Peradze, Superconformal invariance and the geography of four manifolds, Commun. Math. Phys. 205 (1999) 691 [hep-th/9812055] [INSPIRE].

[59] J.P. Serre, A course in arithmetic, Grad. Texts Math. 7, Springer, New York U.S.A., (1973).

[60] D. Zagier, Introduction to modular forms, in From number theory to physics, Springer, Berlin Germany, (1992), pg. 238.

[61] J.H. Bruinier, G. van der Geer, G. Harder and D. Zagier, The 1-2-3 of modular forms, Springer-Verlag, Berlin Heidelberg Germany, (2008).

[62] M.-F. Vignéras, Séries thêta des formes quadratiques indéfinies (in French), Lect. Notes Math. 627 (1977) 227. 